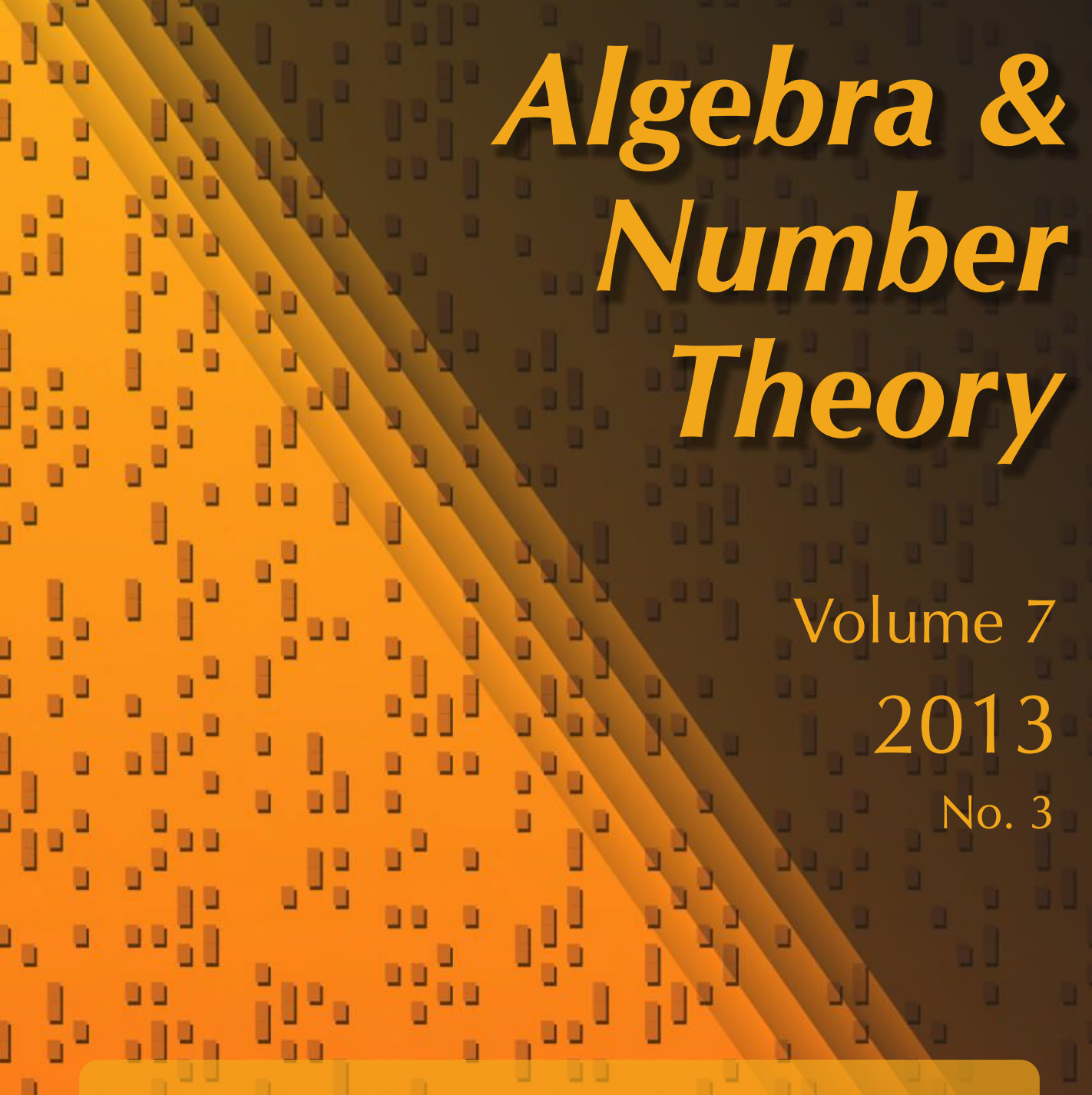

Ekedahl-Oort strata of hyperelliptic curves in characteristic 2

Arsen Elkin and Rachel Pries

\lrcorner

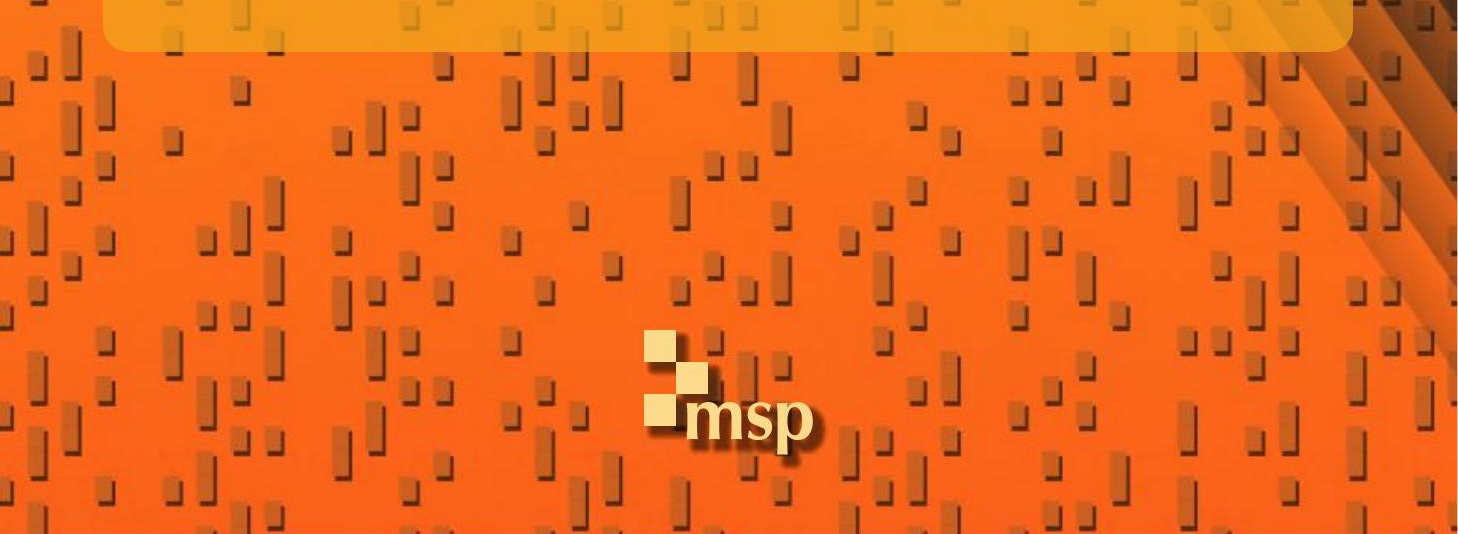




\title{
Ekedahl-Oort strata of hyperelliptic curves in characteristic 2
}

\author{
Arsen Elkin and Rachel Pries
}

\begin{abstract}
Suppose $X$ is a hyperelliptic curve of genus $g$ defined over an algebraically closed field $k$ of characteristic $p=2$. We prove that the de Rham cohomology of $X$ decomposes into pieces indexed by the branch points of the hyperelliptic cover. This allows us to compute the isomorphism class of the 2-torsion group scheme $J_{X}$ [2] of the Jacobian of $X$ in terms of the Ekedahl-Oort type. The interesting feature is that $J_{X}[2]$ depends only on some discrete invariants of $X$, namely, on the ramification invariants associated with the branch points. We give a complete classification of the group schemes that occur as the 2-torsion group schemes of Jacobians of hyperelliptic $k$-curves of arbitrary genus, showing that only relatively few of the possible group schemes actually do occur.
\end{abstract}

\section{Introduction}

Suppose $k$ is an algebraically closed field of characteristic $p>0$. There are several important stratifications of the moduli space $\mathscr{A}_{g}$ of principally polarized abelian varieties of dimension $g$ defined over $k$, including the Ekedahl-Oort stratification. The Ekedahl-Oort type characterizes the $p$-torsion group scheme of the corresponding abelian varieties and, in particular, determines invariants of the group scheme such as the $p$-rank and $a$-number. It is defined by the interaction between the Frobenius $F$ and Verschiebung $V$ operators on the $p$-torsion group scheme. Very little is known about how the Ekedahl-Oort strata intersect the Torelli locus of Jacobians of curves. In particular, one would like to know which group schemes occur as the $p$-torsion $J_{X}[p]$ of the Jacobian $J_{X}$ of a curve $X$ of genus $g$.

In this paper, we completely answer this question for hyperelliptic $k$-curves $X$ of arbitrary genus when $k$ has characteristic $p=2$, a case that is amenable to calculation because of the confluence of hyperelliptic and Artin-Schreier properties. We first prove a decomposition result about the structure of $\mathrm{H}_{\mathrm{dR}}^{1}(X)$ as a module under the

Elkin is partially supported by the Marie Curie Incoming International Fellowship PIIF-GA-2009236606. Pries is partially supported by National Science Foundation grant DMS-11-01712.

MSC2010: primary 11G20; secondary 14K15, 14L15, 14H40, 14F40, $11 \mathrm{G} 10$.

Keywords: curve, hyperelliptic, Artin-Schreier, Jacobian, $p$-torsion, $a$-number, group scheme, de Rham cohomology, Ekedahl-Oort strata. 
actions of $F$ and $V$, where the pieces of the decomposition are indexed by the branch points of the hyperelliptic cover. This is the only decomposition result about the de Rham cohomology of Artin-Schreier curves that we know of, though the action of $V$ on $\mathrm{H}^{0}\left(X, \Omega^{1}\right)$ and the action of $F$ on $\mathrm{H}^{1}(X, \mathcal{O})$ have been studied for ArtinSchreier curves under less restrictive hypotheses [Madden 1978; Sullivan 1975].

The second result of this paper is a complete classification of the isomorphism classes of group schemes that occur as the 2-torsion group scheme $J_{X}$ [2] for a hyperelliptic $k$-curve $X$ of arbitrary genus when $\operatorname{char}(k)=2$. The group schemes that occur decompose into pieces indexed by the branch points of the hyperelliptic cover, and we determine the Ekedahl-Oort types of these pieces. In particular, we determine which $a$-numbers occur for the 2-torsion group schemes of hyperelliptic $k$-curves of arbitrary genus when $\operatorname{char}(k)=2$. Before describing the result precisely, we note that it shows that the group scheme $J_{X}[2]$ depends only on some discrete invariants of $X$ and not on the location of the branch points or the equation of the hyperelliptic cover. This is in sharp contrast to the case of hyperelliptic curves in odd characteristic $p$, where even the $p$-rank depends on the location of the branch points [Yui 1978].

Notation 1.1. Suppose $k$ is an algebraically closed field of characteristic $p=2$. Let $X$ be a $k$-curve of genus $g$ that is hyperelliptic, in other words, for which there exists a degree two cover $\pi: X \rightarrow \mathbb{P}^{1}$. Let $B \subset \mathbb{P}^{1}(k)$ denote the set of branch points of $\pi$, and let $r:=\# B-1$. After a fractional linear transformation, one may suppose that $0 \in B$ and $\infty \notin B$.

For $\alpha \in B$, the ramification invariant $d_{\alpha}$ is the largest integer for which the higher ramification group of $\pi$ above $\alpha$ is nontrivial. By [Stichtenoth 2009, Proposition III.7.8], $d_{\alpha}$ is odd. Let $c_{\alpha}:=\left(d_{\alpha}-1\right) / 2$, and let $x_{\alpha}:=(x-\alpha)^{-1}$.

The cover $\pi$ is given by an affine equation of the form $y^{2}-y=f(x)$ for some nonconstant rational function $f(x) \in k(x)$. After a change of variables of the form $y \mapsto y+\epsilon$, one may suppose the partial fraction decomposition of $f(x)$ has the form

$$
f(x)=\sum_{\alpha \in B} f_{\alpha}\left(x_{\alpha}\right),
$$

where $f_{\alpha}(x) \in x k\left[x^{2}\right]$ is a polynomial of degree $d_{\alpha}$ containing no monomials of even exponent. In particular, the divisor of poles of $f(x)$ on $\mathbb{P}^{1}$ has the form

$$
\operatorname{div}_{\infty}(f(x))=\sum_{\alpha \in B} d_{\alpha} \alpha
$$

By the Riemann-Hurwitz formula [Serre 1968, IV, Proposition 4], the genus $g$ of $X$ satisfies

$$
2 g+2=\sum_{\alpha \in B}\left(d_{\alpha}+1\right)
$$


Recall that the 2-rank of (the Jacobian of) the $k$-curve $X$ is $\operatorname{dim}_{\mathbb{F}_{2}} \operatorname{Hom}\left(\mu_{2}, J_{X}[2]\right)$, where $\mu_{2}$ is the kernel of Frobenius on $\mathbb{G}_{m}$. By the Deuring-Shafarevich formula [Subrao 1975, Theorem 4.2; Crew 1984, Corollary 1.8], the 2-rank of $X$ is $r$. Note that $g=r+\sum_{\alpha \in B} c_{\alpha}$. The implication of these formulas is that, for a given genus $g$ (and 2-rank $r$ ), there is an additional discrete invariant of the hyperelliptic $k$-curve $X$, namely, a partition of $2 g+2$ into $r+1$ positive even integers $d_{\alpha}+1$. In Section 5a, we show that the Ekedahl-Oort type of $X$ depends only on this discrete invariant.

Theorem 1.2. Suppose $X$ is a hyperelliptic curve defined over an algebraically closed field $k$ of characteristic 2 with affine equation $y^{2}-y=f(x)$, branch locus $B$, and polynomials $f_{\alpha}$ for $\alpha \in B$ as described in Notation 1.1. For $\alpha \in B$, consider the Artin-Schreier $k$-curve $Y_{\alpha}$ with affine equation $y^{2}-y=f_{\alpha}(x)$. Let $E$ be an ordinary elliptic $k$-curve. As a module under the actions of Frobenius $F$ and Verschiebung $V$, the de Rham cohomology of $X$ decomposes as

$$
\mathrm{H}_{\mathrm{dR}}^{1}(X) \cong \mathrm{H}_{\mathrm{dR}}^{1}(E)^{\# B-1} \oplus \bigoplus_{\alpha \in B} \mathrm{H}_{\mathrm{dR}}^{1}\left(Y_{\alpha}\right) .
$$

As an application of Theorem 1.2, we give a complete classification of the Ekedahl-Oort types that occur for hyperelliptic $k$-curves. Recall that the 2-torsion group scheme $J_{X}[2]$ of the Jacobian of a $k$-curve is a polarized $\mathrm{BT}_{1}$ group scheme over $k$ (short for polarized Barsotti-Tate truncated level-1 group scheme) and that the isomorphism class of a $\mathrm{BT}_{1}$ group scheme determines and is determined by its Ekedahl-Oort type; see Section 2 for more details. For $p=2$ and a natural number $c$, let $G_{c}$ be the polarized $\mathrm{BT}_{1}$ group scheme of rank $p^{2 c}$ with Ekedahl-Oort type $[0,1,1,2,2, \ldots,\lfloor c / 2\rfloor]$. For example, $G_{1}$ is the 2-torsion group scheme of a supersingular elliptic $k$-curve. The group scheme $G_{2}$ occurs as the 2-torsion of a supersingular nonsuperspecial abelian surface over $k$. The group scheme $G_{c}$ is not necessarily indecomposable. More explanation about $G_{c}$ is given in Sections $2 \mathrm{c}$ and $5 b$.

Before stating the classification result, we note that it also includes a complete description of which $a$-numbers occur for the Jacobians of hyperelliptic $k$-curves. Recall that the $a$-number of $X$ is defined as $a_{X}:=\operatorname{dim}_{k} \operatorname{Hom}\left(\alpha_{2}, J_{X}[2]\right)$, where $\alpha_{2}$ is the kernel of Frobenius on $\mathbb{G}_{a}$.

Theorem 1.3. Let $X$ be a hyperelliptic $k$-curve with affine equation $y^{2}-y=f(x)$ defined over an algebraically closed field of characteristic 2 as described in Notation 1.1. Then the 2-torsion group scheme of the Jacobian variety of $X$ is

$$
J_{X}[2] \simeq\left(\mathbb{Z} / 2 \oplus \mu_{2}\right)^{r} \oplus \bigoplus_{\alpha \in B} G_{c_{\alpha}}
$$

and the a-number of $X$ is $a_{X}=\left(g+1-\#\left\{\alpha \in B \mid d_{\alpha} \equiv 1 \bmod 4\right\}\right) / 2$. 
Theorem 1.3 is stated without proof in [van der Geer 1999, 3.2] for the special case when $f(x) \in k[x]$, that is, $r=0$. There are two interesting things about Theorem 1.3. First, it shows that the Ekedahl-Oort type of $X: y^{2}-y=f(x)$ depends only on the orders of the poles of $f(x)$. This is in sharp contrast to the case of hyperelliptic curves in odd characteristic $p$, where even the $p$-rank depends on $f(x)$ and the location of the branch points [Yui 1978]. Similarly, it differs from the results of [Bouw 2001; Elkin 2011; Johnston 2007], all of which give bounds for the $p$-rank and $a$-number of various kinds of curves that depend strongly on the coefficients of their equations. Likewise, preliminary calculations indicate that it is in contrast to the situation for Artin-Schreier curves in odd characteristic.

Secondly, Theorem 1.3 is interesting because it shows that most of the possibilities for the 2-torsion group scheme of an abelian variety over $k$ do not occur for Jacobians of hyperelliptic $k$-curves when $\operatorname{char}(k)=2$. Specifically, there are $2^{g}$ possibilities for the 2-torsion group scheme of a $g$-dimensional abelian variety over $k$. We determine a subset of these of cardinality equal to the number $P(g+1)$ of partitions of $g+1$ and prove that the group schemes in this subset are exactly those that occur as the 2-torsion $J_{X}[2]$ for a hyperelliptic $k$-curve $X$ of genus $g$. Recall [Hardy and Ramanujan 1918] that $P(g+1)$ grows asymptotically like $e^{\pi \sqrt{2(g+1) / 3}} /(4 \sqrt{3}(g+1))$ as $g$ goes to infinity. Also, Theorem 1.3 gives the nontrivial bounds $(g-r) / 2 \leq a_{X} \leq(g+1) / 2$ for the $a$-number.

An earlier nonexistence result of this type is due to Ekedahl [1987], who proved that a curve $X$ of genus $g>p(p-1) / 2$ in characteristic $p>0$ cannot be superspecial and thus $a_{X}<g$. There are also other recent results about Newton polygons of hyperelliptic (that is, Artin-Schreier) curves in characteristic 2, including several nonexistence results [Blache 2012; Scholten and Zhu 2002]. In addition, there are closed formulas for the number of hyperelliptic curves of genus 3 with given 2-rank over each finite field of characteristic 2 [Nart and Sadornil 2004].

Here is an outline of this paper. Section 2 contains notation and background. Results on $\mathrm{H}^{0}\left(X, \Omega^{1}\right)$ and the $a$-number are in Section 3. Theorem 1.2 is with the material on the de Rham cohomology in Section 4. Section 5 contains the results about the Ekedahl-Oort type, including Theorem 1.3.

\section{Background}

In this paper, all objects are defined over an algebraically closed field $k$ of characteristic $p>0$, and all curves are smooth, projective, and connected. This section includes background on $p$-torsion group schemes, Ekedahl-Oort types, the de Rham cohomology, and Frobenius and Verschiebung.

2a. The p-torsion group scheme. Suppose $A$ is a principally polarized abelian variety of dimension $g$ defined over $k$. For example, $A$ could be the Jacobian of a 
$k$-curve of genus $g$. Consider the multiplication-by- $p$ morphism $[p]: A \rightarrow A$ that is a finite flat morphism of degree $p^{2 g}$. It factors as $[p]=V \circ F$. Here $F: A \rightarrow A^{(p)}$ is the relative Frobenius morphism coming from the $p$-power map on the structure sheaf; it is purely inseparable of degree $p^{g}$. Furthermore, $V: A^{(p)} \rightarrow A$ is the Verschiebung morphism.

The $p$-torsion group scheme of $A$, denoted $A[p]$, is the kernel of $[p]$. It is a finite commutative group scheme annihilated by $p$, again having morphisms $F$ and $V$. By [Oort 2001, 9.5], the $p$-torsion group scheme $A[p]$ is a polarized $\mathrm{BT}_{1}$ group scheme over $k$ (short for polarized Barsotti-Tate truncated level-1 group scheme) as defined in [Oort 2001, 2.1, 9.2]. The rank of $A[p]$ is $p^{2 g}$.

We now give a brief summary of the classification [Oort 2001, Theorems 9.4 and 12.3] of polarized $\mathrm{BT}_{1}$ group schemes over $k$ in terms of Dieudonné modules and Ekedahl-Oort type; other useful references are [Kraft 1975] (without polarization) and [Moonen 2001] (for $p \geq 3$ ).

2b. The Dieudonné module and polarizations. It is useful to describe the group scheme $A[p]$ using (the modulo $p$ reduction of) the covariant Dieudonné module [Oort 2001, 15.3]. This is the dual of the contravariant theory found in [Demazure 1972]. In brief, consider the noncommutative ring $\mathbb{E}=k[F, V]$ generated by semilinear operators $F$ and $V$ with the relations $F V=V F=0$ and $F \lambda=\lambda^{p} F$ and $\lambda V=V \lambda^{p}$ for all $\lambda \in k$. Let $\mathbb{E}(A, B)$ denote the left ideal $\mathbb{E} A+\mathbb{E} B$ of $\mathbb{E}$ generated by $A$ and $B$. A deep result is that the Dieudonné functor $D$ gives an equivalence of categories between $\mathrm{BT}_{1}$ group schemes over $k$ (with rank $p^{2 g}$ ) and finite left $\mathbb{E}$-modules (having dimension $2 g$ as a $k$-vector space). We use the notation $D(\mathbb{G})$ to denote the Dieudonné module of $\mathbb{G}$. For example, the Dieudonné module of the $p$-torsion group scheme of an ordinary elliptic curve is $D\left(\mathbb{Z} / p \oplus \mu_{p}\right) \simeq$ $\mathbb{E} / \mathbb{E}(F, 1-V) \oplus \mathbb{E} / \mathbb{E}(V, 1-F)$ [Goren 2002, Examples A.5.1 and A.5.3].

The polarization of $A$ induces a symmetry on $A[p]$ as defined in [Oort 2001, 5.1], namely, an antisymmetric isomorphism from $A[p]$ to the Cartier dual group scheme $A[p]^{\text {dual }}$ of $A[p]$. Unfortunately, in characteristic 2 , there may be antisymmetric morphisms $A[p] \rightarrow A[p]^{\text {dual }}$ that do not come from a polarization. Luckily, this issue can be resolved by defining a polarization on $A[p]$ in terms of a nondegenerate alternating pairing on $D(A[p])$ [Oort 2001, 9.2, 9.5, 12.2].

2c. The Ekedahl-Oort type. As in [Oort 2001, Sections 5 and 9], the isomorphism type of a $\mathrm{BT}_{1}$ group scheme $\mathbb{G}$ over $k$ can be encapsulated into combinatorial data. If $\mathbb{G}$ is symmetric with rank $p^{2 g}$, then there is a final filtration $N_{1} \subset N_{2} \subset \cdots \subset N_{2 g}$ of $\mathbb{G}$ as a $k$-vector space that is stable under the action of $V$ and $F^{-1}$ such that $i=\operatorname{dim}\left(N_{i}\right)$ [Oort 2001, 5.4]. If $w$ is a word in $V$ and $F^{-1}$, then $w D(\mathbb{G})$ is an object in the filtration; in particular, $N_{g}=V D(\mathbb{G})=F^{-1}(0)$. 
The Ekedahl-Oort type of $\mathbb{G}$, also called the final type, is $v=\left[v_{1}, \ldots, v_{g}\right]$, where $v_{i}=\operatorname{dim}\left(V\left(N_{i}\right)\right)$. The Ekedahl-Oort type of $\mathbb{G}$ does not depend on the choice of a final filtration. There is a restriction $v_{i} \leq v_{i+1} \leq v_{i}+1$ on the final type. There are $2^{g}$ Ekedahl-Oort types of length $g$ since all sequences satisfying this restriction occur. By [Oort 2001, 9.4, 12.3], there are bijections between (i) Ekedahl-Oort types of length $g$, (ii) polarized $\mathrm{BT}_{1}$ group schemes over $k$ of rank $p^{2 g}$, and (iii) principal quasipolarized Dieudonné modules of dimension $2 g$ over $k$.

2d. The p-rank and a-number. Two invariants of (the $p$-torsion of) an abelian variety are the $p$-rank and $a$-number. The $p$-rank of $A$ is $r=\operatorname{dim}_{\mathbb{F}_{p}} \operatorname{Hom}\left(\mu_{p}, A[p]\right)$, where $\mu_{p}$ is the kernel of Frobenius on $\mathbb{G}_{m}$. Then $p^{r}$ is the cardinality of $A[p](k)$. The $a$-number of $A$ is $a=\operatorname{dim}_{k} \operatorname{Hom}\left(\alpha_{p}, A[p]\right)$, where $\alpha_{p}$ is the kernel of Frobenius on $\mathbb{G}_{a}$. It is well known that $0 \leq f \leq g$ and $1 \leq a+f \leq g$. The $p$-rank of $A[p]$ equals the dimension of $V^{g} D(\mathbb{G})$. The $a$-number of $A[p]$ equals $g-\operatorname{dim}\left(V^{2} D(\mathbb{G})\right)$ [Li and Oort 1998, 5.2.8]. The $p$-rank equals $\max \left\{i \mid v_{i}=i\right\}$, and the $a$-number equals $g-v_{g}$.

2e. The de Rham cohomology. Suppose $X$ is a $k$-curve of genus $g$, and recall the definition of the noncommutative ring $\mathbb{E}=k[F, V]$ from Section 2b. By [Oda 1969, Section 5], there is an isomorphism of $\mathbb{E}$-modules between the Dieudonné module of the $p$-torsion group scheme $J_{X}[p]$ and the de Rham cohomology group $\mathrm{H}_{\mathrm{dR}}^{1}(X)$. In particular, $\operatorname{ker}(F)=\mathrm{H}^{0}\left(X, \Omega^{1}\right)=\operatorname{im}(V)$. Recall that $\operatorname{dim}_{k} \mathrm{H}_{\mathrm{dR}}^{1}(X)=2 g$.

In [Oda 1969, Section 5], there is the following description of $\mathrm{H}_{\mathrm{dR}}^{1}(X)$. Let $u=\left\{U_{i}\right\}$ be a covering of $X$ by affine open subvarieties, and let $U_{i j}:=U_{i} \cap U_{j}$ and $U_{i j k}:=U_{i} \cap U_{j} \cap U_{k}$. For a sheaf $\mathscr{F}$ on $X$, let

$$
\begin{aligned}
& \mathrm{C}^{0}(\mathcal{U}, \mathscr{F}):=\left\{\kappa=\left(\kappa_{i}\right)_{i} \mid \kappa_{i} \in \Gamma\left(U_{i}, \mathscr{F}\right)\right\}, \\
& \mathrm{C}^{1}(\mathcal{U}, \mathscr{F}):=\left\{\phi=\left(\phi_{i j}\right)_{i<j} \mid \phi_{i j} \in \Gamma\left(U_{i j}, \mathscr{F}\right)\right\}, \\
& \mathrm{C}^{2}(\mathcal{U}, \mathscr{F}):=\left\{\psi=\left(\psi_{i j k}\right)_{i<j<k} \mid \psi_{i j k} \in \Gamma\left(U_{i j k}, \mathscr{F}\right)\right\} .
\end{aligned}
$$

For convenience, let $\phi_{i i}:=0$ for any $\phi \in \mathrm{C}^{1}(\mathcal{U}, \mathscr{F})$. There are coboundary operators $\delta: \mathrm{C}^{0}(\mathcal{U}, \mathscr{F}) \rightarrow \mathrm{C}^{1}(\mathcal{U}, \mathscr{F})$ defined by $(\delta \kappa)_{i<j}=\kappa_{i}-\kappa_{j}$ and $\delta: \mathrm{C}^{1}(\mathcal{U}, \mathscr{F}) \rightarrow \mathrm{C}^{2}(U, \mathscr{F})$ by $(\delta \phi)_{i<j<k}=\phi_{i j}-\phi_{i k}+\phi_{j k}$. All other maps are applied to $\mathrm{C}^{m}(\mathcal{U}, \mathscr{F})$ elementwise, for example, $(F \phi)_{i}:=F \phi_{i}$. As expected, $\delta^{2}=0$.

The de Rham cocycles are defined by

$$
\mathrm{Z}_{\mathrm{dR}}^{1}(\mathcal{(}):=\left\{(\phi, \omega) \in \mathrm{C}^{1}(\mathcal{U}, \mathcal{O}) \times \mathrm{C}^{0}\left(\mathcal{U}, \Omega^{1}\right) \mid \delta \phi=0, d \phi=\delta \omega\right\},
$$

that is, $\phi_{i j}-\phi_{i k}+\phi_{j k}=0$ and $d \phi_{i j}=\omega_{i}-\omega_{j}$ for all indices $i<j<k$. The de Rham coboundaries are defined by

$$
\mathrm{B}_{\mathrm{dR}}^{1}(U):=\left\{(\delta \kappa, d \kappa) \in \mathrm{Z}_{\mathrm{dR}}^{1}(u) \mid \kappa \in C^{0}(U, \mathcal{O})\right\} .
$$


Finally,

$$
\mathrm{H}_{\mathrm{dR}}^{1}(X) \cong \mathrm{H}_{\mathrm{dR}}^{1}(u):=\mathrm{Z}_{\mathrm{dR}}^{1}(u) / \mathrm{B}_{\mathrm{dR}}^{1}(u) .
$$

There is an injective homomorphism $\lambda: \mathrm{H}^{0}\left(X, \Omega^{1}\right) \rightarrow \mathrm{H}_{\mathrm{dR}}^{1}(X)$ denoted informally by $\omega \mapsto(0, \omega)$, where the second coordinate is defined by $\omega_{i}=\left.\omega\right|_{U_{i}}$. This map is well-defined since $d(0)=\left.\omega\right|_{U_{i}}-\left.\omega\right|_{U_{j}}=(\delta \omega)_{i<j}$. It is injective because if $\left(0, \omega_{1}\right) \equiv\left(0, \omega_{2}\right) \bmod \mathrm{B}_{\mathrm{dR}}^{1}(\mathcal{U})$, then $\omega_{1}-\omega_{2}=d \kappa$, where $\kappa \in C^{0}(\mathcal{U}, \mathcal{O})$ is such that $\delta \kappa=0$; thus, $\kappa \in \mathrm{H}^{0}(\mathcal{U}, \mathcal{O}) \simeq k$ is a constant function on $X$, and so $\omega_{1}-\omega_{2}=0$.

There is another homomorphism $\gamma: \mathrm{H}_{\mathrm{dR}}^{1}(X) \rightarrow \mathrm{H}^{1}(X, \mathcal{O})$ sending the cohomology class of $(\phi, \omega)$ to the cohomology class of $\phi$. The choice of cocycle $(\phi, \omega)$ does not matter since the coboundary conditions on $\mathrm{H}_{\mathrm{dR}}^{1}(X)$ and $\mathrm{H}^{1}(X, O)$ are compatible. The homomorphisms $\lambda$ and $\gamma$ fit into a short exact sequence

$$
0 \rightarrow \mathrm{H}^{0}\left(X, \Omega^{1}\right) \stackrel{\lambda}{\rightarrow} \mathrm{H}_{\mathrm{dR}}^{1}(X) \stackrel{\gamma}{\rightarrow} \mathrm{H}^{1}(X, \mathcal{O}) \rightarrow 0
$$

of $k$-vector spaces. In Sections $4 \mathrm{~d}$ and $4 \mathrm{f}$, we construct a suitable section $\sigma$ : $\mathrm{H}^{1}(X, O) \rightarrow \mathrm{H}_{\mathrm{dR}}^{1}(X)$ of $\gamma$ when $X$ is a hyperelliptic $k$-curve with $\operatorname{char}(k)=2$.

2f. Frobenius and Verschiebung. The Cartier operator $\mathscr{b}$ on the sheaf $\Omega^{1}$ is defined in [Cartier 1957]. Its three principal properties are that it annihilates exact differentials, preserves logarithmic ones, and induces a $p^{-1}$-linear map on $\mathrm{H}^{0}\left(X, \Omega^{1}\right)$. The Cartier operator can be computed as follows. Let $x \in k(X)$ be an element that forms a $p$-basis of $k(X)$ over $k(X)^{p}$, that is, an element such that every $z \in k(X)$ can be written as

$$
z:=z_{0}^{p}+z_{1}^{p} x+\cdots+z_{p-1}^{p} x^{p-1}
$$

for uniquely determined $z_{0}, \ldots, z_{p-1} \in k(X)$. Then

$$
\mathscr{C}(z d x / x):=z_{0} d x / x .
$$

The Frobenius operator $F$ on the structure sheaf $\mathcal{O}$ of $X$ induces a $p$-linear map $F$ on $\mathrm{H}^{1}(X, \mathcal{O})$. By Serre duality, the $k[F]$-module $\mathrm{H}^{1}(X, \mathcal{O})$ is dual to the $k[\mathscr{C}]$-module $\mathrm{H}^{0}\left(X, \Omega^{1}\right)$.

The $p$-linear operator $F$ and the $p^{-1}$-linear operator $V$ are defined on $\mathrm{H}_{\mathrm{dR}}^{1}(X)$ as follows. Let $V(\omega):=\mathscr{C}(\omega)$ and $F(\omega):=0$ for $\omega \in \mathrm{H}^{0}\left(X, \Omega^{1}\right)$ and $V(f):=0$ for $f \in \mathrm{H}^{1}(X, \mathcal{O})$. Then

$F(f, \omega):=(F(f), F(\omega))=\left(f^{p}, 0\right) \quad$ and $\quad V(f, \omega):=(V(f), V(\omega))=(0, \mathscr{C}(\omega))$.

With $\mathbb{E}=k[F, V]$ defined in Section 2c, the short exact sequence (2-1) is an exact sequence of $\mathbb{E}$-modules. However, the section $\sigma$ of (2-1) constructed in Section $4 \mathrm{~d}$ is not a splitting of $\mathbb{E}$-modules. 


\section{Results about regular 1-forms and the $a$-number}

We specialize to the case when the algebraically closed field $k$ has characteristic $p=2$. Consider a hyperelliptic $k$-curve $X$ with affine equation $y^{2}-y=f(x)$ as described in Section 1. For each branch point $\alpha \in B$, recall the definitions of the ramification invariant $d_{\alpha}=2 c_{\alpha}+1$, the function $x_{\alpha}=(x-\alpha)^{-1}$, and the polynomial $f_{\alpha}\left(x_{\alpha}\right)$ appearing in the partial fraction decomposition of $f(x)$. Important facts mentioned in Section 1 are that the genus is determined from the ramification invariants by the formula $2 g+2=\sum_{\alpha \in B}\left(d_{\alpha}+1\right)$ and that the 2-rank of $J_{X}$ equals $r=\# B-1$.

For $\alpha \in B$, let $P_{\alpha}:=\pi^{-1}(\alpha) \in X(k)$ be the ramification point above $\alpha$, and define the divisor $D_{\infty}:=\pi^{-1}(\infty)$ on $X$. Recall that $0 \in B$ and $\infty \notin B$, and let $B_{\infty}:=B \cup\{\infty\}$ and $B^{\prime}:=B-\{0\}$.

3a. The space $\mathbf{H}^{\mathbf{0}}\left(\boldsymbol{X}, \boldsymbol{\Omega}^{\mathbf{1}}\right)$. For an integer $j$ and for $\alpha \in B$, consider the 1-forms

$$
\omega_{\alpha, j}:=x_{\alpha}^{j-1} d x_{\alpha} \quad \text { on } X .
$$

Note that $\omega_{\alpha, j}=-(x-\alpha)^{-j-1} d x$ and if $\alpha \in B^{\prime}$, then $\omega_{\alpha, 0}-\omega_{0,0}=-\alpha d x / x(x-\alpha)$.

For completeness, we prove the next lemma, a variation of a special case of [Sullivan 1975, Lemma 1(c)].

Lemma 3.1. A basis for $\mathrm{H}^{0}\left(X, \Omega^{1}\right)$ is given by the 1 -forms $\omega_{\alpha, j}$ for $\alpha \in B$ and $1 \leq j \leq c_{\alpha}$ and $\omega_{\alpha, 0}-\omega_{0,0}$ for $\alpha \in B^{\prime}$.

Proof. For $\alpha \in B$, we can calculate the following divisors on $X$ : $\operatorname{div}\left(x_{\alpha}\right)=D_{\infty}-2 P_{\alpha}$,

$$
\begin{aligned}
& \operatorname{div}\left(d x_{\alpha}\right)=\left(d_{\alpha}-3\right) P_{\alpha}+\sum_{\beta \in B-\{\alpha\}}\left(d_{\beta}+1\right) P_{\beta}, \\
& \operatorname{div}\left(\omega_{\alpha, j}\right)=2\left(c_{\alpha}-j\right) P_{\alpha}+(j-1) D_{\infty}+\sum_{\beta \in B-\{\alpha\}}\left(d_{\beta}+1\right) P_{\beta} .
\end{aligned}
$$

Thus, $\omega_{\alpha, j}$ is regular for $1 \leq j \leq c_{\alpha}$, and $\left(\omega_{\alpha, 0}-\omega_{0,0}\right)$ is regular for $\alpha \in B^{\prime}$ since

$$
\operatorname{div}\left(\omega_{\alpha, 0}-\omega_{0,0}\right)=2 c_{\alpha} P_{\alpha}+2 c_{0} P_{0}+\sum_{\beta \in B-\{0, \alpha\}}\left(d_{\beta}+1\right) P_{\beta} .
$$

This set of regular differentials of $X$ is linearly independent because the corresponding set of divisors is linearly independent over $\mathbb{Z}$. It forms a basis since the set has cardinality $r+\sum_{\alpha \in B} c_{\alpha}=g$.

Lemma 3.2. If $\alpha \in B$, then

$$
\mathscr{C}\left(\omega_{\alpha, j}\right)= \begin{cases}\omega_{\alpha, j / 2} & \text { if } j \text { is even, } \\ 0 & \text { if } j \text { is odd }\end{cases}
$$

In particular, $\mathscr{C}\left(\omega_{\alpha, 0}-\omega_{0,0}\right)=\omega_{\alpha, 0}-\omega_{0,0}$ for all $\alpha \in B^{\prime}$. 
Proof. Using the properties of the Cartier operator found in Section 2f, one computes when $j$ is even that

$$
\mathscr{C}\left(x_{\alpha}^{j-1} d x_{\alpha}\right)=x_{\alpha}^{j / 2} \mathscr{C}\left(d x_{\alpha} / x_{\alpha}\right)=x_{\alpha}^{j / 2-1} d x_{\alpha}
$$

and when $j$ is odd that

$$
\mathscr{C}\left(x_{\alpha}^{j-1} d x_{\alpha}\right)=x_{\alpha}^{(j-1) / 2} \mathscr{C}\left(d x_{\alpha}\right)=0 .
$$

Let $W_{\alpha, \mathrm{ss}}^{\prime}:=\left\langle\omega_{\alpha, 0}-\omega_{0,0}\right\rangle$ for $\alpha \in B^{\prime}$, and let $W_{\alpha, \text { nil }}^{\prime}:=\left\langle\omega_{\alpha, j} \mid 1 \leq j \leq c_{\alpha}\right\rangle$ for $\alpha \in B$, where $\langle\cdot\rangle$ denotes the $k$-span. These subspaces are invariant under the Cartier operator by Lemma 3.2.

Lemma 3.3. The subspaces $W_{\alpha, \mathrm{ss}}^{\prime}$ and $W_{\alpha, \mathrm{nil}}^{\prime}$ of $\mathrm{H}^{0}\left(X, \Omega^{1}\right)$ are stable under the action of Verschiebung for each $\alpha \in B$. There is an isomorphism of $V$-modules

$$
\mathrm{H}^{0}\left(X, \Omega^{1}\right) \simeq \bigoplus_{\alpha \in B^{\prime}} W_{\alpha, \mathrm{ss}}^{\prime} \oplus \bigoplus_{\alpha \in B} W_{\alpha, \text { nil }}^{\prime}
$$

Proof. This follows immediately from Lemmas 3.1 and 3.2.

3b. Application: The a-number.

Proposition 3.4. Let $X$ be a hyperelliptic $k$-curve with affine equation $y^{2}-y=f(x)$ as described in Notation 1.1. If $\operatorname{div}_{\infty}(f(x))=\sum_{\alpha \in B} d_{\alpha} \alpha$ is the divisor of poles of $f(x)$ on $\mathbb{P}^{1}$, then the a-number of $X$ is

$$
a_{X}=\frac{g+1-\#\left\{\alpha \in B \mid d_{\alpha} \equiv 1 \bmod 4\right\}}{2} .
$$

Proof. The $a$-number of $\mathbb{G}=J_{X}[2]$ is $a_{X}=g-\operatorname{dim}\left(V^{2} D(\mathbb{G})\right)$ [Li and Oort 1998, 5.2.8]. The action of $V$ on $V D(\mathbb{G})$ is the same as the action of the Cartier operator $\mathscr{C}$ on $\mathrm{H}^{0}\left(X, \Omega^{1}\right)$. So $a_{X}$ equals the dimension of the kernel of $\mathscr{C}$ on $\mathrm{H}^{0}\left(X, \Omega^{1}\right)$. By Lemma 3.2, the kernel of $\mathscr{C}$ on $\mathrm{H}^{0}\left(X, \Omega^{1}\right)$ is spanned by $\omega_{\alpha, j}$ for $\alpha \in B$ and $j$ odd with $1 \leq j \leq c_{\alpha}=\left(d_{\alpha}-1\right) / 2$. Thus, the contribution to the $a$-number from each $\alpha \in B$ is $\left\lfloor\left(d_{\alpha}+1\right) / 4\right\rfloor$. In other words, if $d_{\alpha} \equiv 1 \bmod 4$, the contribution is $\left(d_{\alpha}-1\right) / 4$, and if $d_{\alpha} \equiv 3 \bmod 4$, the contribution is $\left(d_{\alpha}+1\right) / 4$. Since $g+1=\sum_{\alpha \in B}\left(d_{\alpha}+1\right) / 2$, this yields

$$
2 a_{X}=(g+1)-\#\left\{\alpha \in B \mid d_{\alpha} \equiv 1 \bmod 4\right\} .
$$

3c. Examples with large p-rank. Let $A$ be a principally polarized abelian variety over $k$ with dimension $g$ and $p$-rank $r$. If $r=g$, then $A[p] \simeq\left(\mathbb{Z} / p \oplus \mu_{p}\right)^{g}$ and the $a$-number is $a=0$. If $r=g-1$, then $A[p] \simeq\left(\mathbb{Z} / p \oplus \mu_{p}\right)^{g-1} \oplus E[p]$, where $E$ is a supersingular elliptic curve and the $a$-number is $a=1$. So the first case where $A[p]$ and $a$ are not determined by the $p$-rank is when $r=g-2$. 
Example 3.5. Let $g \geq 2$. There are two possibilities for the $p$-torsion group scheme of a principally polarized abelian variety over $k$ with dimension $g$ and $p$-rank $g-2$. When $p=2$, both of these occur as the 2-torsion group scheme $J_{X}$ [2] of the Jacobian of a hyperelliptic $k$-curve $X$ of genus $g$.

Proof. If $A$ is a principally polarized abelian variety over $k$ with dimension $g$ and $p$-rank $g-2$, then $A[p] \simeq\left(\mu_{p} \oplus \mathbb{Z} / p\right)^{g-2} \oplus \mathbb{G}$, where $\mathbb{G}$ is isomorphic to the $p$-torsion group scheme of an abelian surface $Z$ with $p$-rank 0 . The abelian surface can be superspecial or merely supersingular. In the superspecial case, $\mathbb{G}=\left(G_{1}\right)^{2}$, where $G_{1}$ denotes the $p$-torsion group scheme of a supersingular elliptic $k$-curve; in the merely supersingular case, we denote the group scheme $G_{2}$; see [Goren 2002, Example A.3.15; Pries 2008, Example 2.3] for a complete description of $G_{2}$.

To prove the second claim, consider the two possibilities for a partition of $2 g+2$ into $r+1=g-1$ even integers: (A) $\{2,2, \ldots, 2,4,4\}$ or (B) $\{2,2, \ldots, 2,2,6\}$. In case (A), consider $f(x) \in k(x)$ with $g-1$ poles such that 0 and 1 are poles of order 3 and the other poles are simple. In case (B), consider $f(x) \in k(x)$ with $g-1$ poles such that 0 is a pole of order 5 and the other poles are simple. The kernel of the Cartier operator on $\mathrm{H}^{0}\left(X, \Omega^{1}\right)$ is spanned by $d x / x^{2}$ and $d x /(x-1)^{2}$ in case (A) and by $d x / x^{2}$ in case (B). Thus, the $a$-number equals 2 in case (A) and equals 1 in case (B). In both cases, this completely determines the group scheme. Namely, the group scheme $J_{X}[2]$ is isomorphic to $\left(\mathbb{Z} / 2 \oplus \mu_{2}\right)^{g-2} \oplus\left(G_{1}\right)^{2}$ in case (A) and to $\left(\mathbb{Z} / 2 \oplus \mu_{2}\right)^{g-2} \oplus G_{2}$ in case (B).

For $g \geq 3$ and $r \leq g-3$, the action of $V$ on $\mathrm{H}^{0}\left(X, \Omega^{1}\right)$ (and, in particular, the value of the $a$-number) is not sufficient to determine the isomorphism class of the group scheme $J_{X}$ [2]. To determine this group scheme, in the next section we study the $\mathbb{E}$-module structure of $\mathrm{H}_{\mathrm{dR}}^{1}(X)$.

\section{Results on the de Rham cohomology}

4a. An open covering. Let $V^{\prime}=\mathbb{P}^{1}-B_{\infty}$ and $U^{\prime}=\pi^{-1}\left(V^{\prime}\right)=X-\pi^{-1}\left(B_{\infty}\right)$. For $\alpha \in B_{\infty}$, let $V_{\alpha}=V^{\prime} \cup\{\alpha\}$ and $U_{\alpha}:=U^{\prime} \cup\left\{\pi^{-1}(\alpha)\right\}$. Then the collection $\mathcal{U}:=\left\{U_{\alpha} \mid \alpha \in B_{\infty}\right\}$ is a cover of $X$ by open affine subvarieties. By construction, if $\alpha, \beta \in B_{\infty}$ are distinct, then $V_{\alpha \beta}:=V_{\alpha} \cap V_{\beta}=V^{\prime}$ and $U_{\alpha \beta}:=U_{\alpha} \cap U_{\beta}=U^{\prime}$. In particular, the subvarieties $U_{\alpha \beta}$ do not depend on the choice of $\alpha$ and $\beta$.

For a sheaf $\mathscr{F}$, let $\mathrm{Z}^{1}(U, \mathscr{F})$ and $\mathrm{B}^{1}(\mathcal{U}, \mathscr{F})$ denote the closed cocycles and coboundaries of $\mathscr{F}$ with respect to $U$. Recall the definition of the noncommutative ring $\mathbb{E}=k[F, V]$ and the notation about $\mathrm{H}_{\mathrm{dR}}^{1}(X)$ from Section 2e. In this section, we compute $\mathrm{H}^{1}(X, \mathcal{O}) \simeq \mathrm{H}^{1}(u, O)$ and $\mathrm{H}_{\mathrm{dR}}^{1}(X) \simeq \mathrm{H}_{\mathrm{dR}}^{1}(\mathcal{U})$ with respect to the open covering $U$ of $X$.

4b. Defining components. Given a sheaf $\mathscr{F}$ and a cocycle $\phi \in \mathrm{Z}^{1}(U, \mathscr{F})$, consider its components $\phi_{\alpha \infty} \in \Gamma\left(U^{\prime}, \mathscr{F}\right)$ for $\alpha \in B$. We call $\left\{\phi_{\alpha \infty} \mid \alpha \in B\right\}$ the set of 
defining components of $\phi$. The reason is that the remaining components of $\phi$ are determined by the coboundary condition $\phi_{\alpha \beta}=\phi_{\alpha \infty}-\phi_{\beta \infty}$. A collection of sections $\left\{\phi_{\alpha \infty} \in \Gamma\left(U^{\prime}, \mathscr{F}\right) \mid \alpha \in B\right\}$ determines a unique closed cocycle $\phi \in \mathrm{Z}^{1}(\mathcal{U}, \mathscr{F})$. Thus,

$$
\mathrm{Z}^{1}(\mathcal{\mathscr { F }}) \cong \bigoplus_{\alpha \in B} \Gamma\left(U^{\prime}, \mathscr{F}\right)
$$

For $\beta \in B$, consider the natural $k$-linear map

$$
\varphi_{\beta}: \Gamma\left(U^{\prime}, \mathcal{O}\right) \rightarrow \mathrm{Z}^{1}(\mathcal{\imath}, \mathrm{O})
$$

whose defining components for $\alpha \in B$ are

$$
\left(\varphi_{\beta}(h)\right)_{\alpha \infty}:= \begin{cases}h & \text { if } \alpha=\beta \\ 0 & \text { otherwise. }\end{cases}
$$

Also, consider the $k$-linear map $\varphi_{\infty}: \Gamma\left(U^{\prime}, \mathcal{O}\right) \rightarrow Z^{1}(u, \mathcal{O})$ defined by

$$
\left(\varphi_{\infty}(h)\right)_{\alpha \infty}:=-h \text { for all } \alpha \in B .
$$

Observe that if $h \in \Gamma\left(U^{\prime}, \mathcal{O}\right)$, then

$$
\sum_{\beta \in B_{\infty}} \varphi_{\beta}(h)=0 .
$$

For $\beta \in B_{\infty}$, consider the natural $k$-linear map

$$
\psi_{\beta}: \Gamma\left(U_{\beta}, \mathcal{O}\right) \rightarrow \mathrm{C}^{0}(\mathcal{\imath}, \mathcal{O})
$$

given for $\alpha \in B_{\infty}$ by

$$
\left(\psi_{\beta}(h)\right)_{\alpha}:= \begin{cases}h & \text { if } \alpha=\beta \\ 0 & \text { otherwise. }\end{cases}
$$

It is straightforward to verify the next lemma.

Lemma 4.1. Suppose $\beta \in B_{\infty}$ and $h \in \Gamma\left(U_{\beta}, \mathcal{O}\right)$ (that is, $h$ is regular at $P_{\beta}$ if $\beta \neq \infty$ and $h$ is regular at the two points in the support of $D_{\infty}$ if $\beta=\infty$ ). Then $\varphi_{\beta}\left(\left.h\right|_{U^{\prime}}\right)=\delta \psi_{\beta}(h)$ is a coboundary.

4c. The space $\mathbf{H}^{\mathbf{1}}(X, O)$. In this section, we find an $F$-module decomposition of $\mathrm{H}^{1}(X, O) \simeq \mathrm{H}^{1}(U, \mathcal{O})$. The results could be deduced from Section 3a using the duality between $\mathrm{H}^{1}(X, \mathcal{O})$ and $\mathrm{H}^{0}\left(X, \Omega^{1}\right)$. Instead, we take a direct approach because an explicit description of $\mathrm{H}^{1}(X, O)$ is helpful for studying $\mathrm{H}_{\mathrm{dR}}^{1}(X)$ in Section $4 \mathrm{f}$.

Lemma 4.2. Write $D_{\infty}=P_{\infty, 1}+P_{\infty, 2}$. Then $\operatorname{ord}_{P_{\infty, 1}}(y)=0$ and $\operatorname{ord}_{P_{\infty, 2}}(y)=s$ for some $s \geq 0$ (possibly after reordering). For $\alpha \in B$ and $j \in \mathbb{Z}$, the divisor of poles 
on $X$ of the function $y x_{\alpha}^{-j}=y(x-\alpha)^{j}$ satisfies

$$
\begin{aligned}
& \operatorname{div}_{\infty}\left(y(x-\alpha)^{j}\right) \\
& \quad=\max \left(d_{\alpha}-2 j, 0\right) P_{\alpha}+\max (j, 0) P_{\infty, 1}+\max (j-s, 0) P_{\infty, 2}+\sum_{\beta \in B-\{\alpha\}} d_{\beta} P_{\beta} .
\end{aligned}
$$

Proof. Recall that $\operatorname{div}_{\infty}(y)=\sum_{\beta \in B} d_{\beta} P_{\beta}$. Note that $\operatorname{ord}_{P_{\infty, i}}(y) \geq 0$ for $i=1,2$ since $\infty \notin B$. If $\operatorname{ord}_{P_{\infty, 2}}(y)>0$, that is, if $y$ has a zero at $P_{\infty, 2}$, then the value of $y$ is one at the Galois conjugate $P_{\infty, 1}$ of $P_{\infty, 2}$. Thus, $y$ cannot have a zero at both points in the support of $D_{\infty}$. The second claim follows from the additional fact that $\operatorname{div}(x-\alpha)=2 P_{\alpha}-D_{\infty}$ for $\alpha \in B$.

Lemma 4.2 implies that $y(x-\alpha)^{j} \in \Gamma\left(U^{\prime}, \mathcal{O}\right)$ for all $\alpha \in B$ and $j \in \mathbb{Z}$.

Lemma 4.3. With notation as above,

(i) $Z^{1}(U, \mathbb{O})=\left\langle\varphi_{\beta}\left((x-\alpha)^{j}\right), \varphi_{\beta}\left(y(x-\alpha)^{j}\right) \mid \alpha, \beta \in B, j \in \mathbb{Z}\right\rangle$, and

(ii) if $\alpha \in B$, then $\left\langle\varphi_{\alpha}\left(y(x-\beta)^{j}\right) \mid j \geq 0\right\rangle=\left\langle\varphi_{\alpha}\left(y(x-\alpha)^{j}\right) \mid j \geq 0\right\rangle$ as subspaces of $Z^{1}(U, \mathcal{O})$ for each $\beta \in B$.

Proof. (i) This is immediate from Equation (4-1) because

$$
\mathrm{Z}^{1}(\mathcal{U}, \mathcal{O})=\bigoplus_{\beta \in B}\left\langle\varphi_{\beta}(h) \mid h \in \Gamma\left(U^{\prime}, \mathcal{O}\right)\right\rangle .
$$

(ii) Both are equal to the subspace $\left\{\varphi_{\alpha}(y h(x)) \mid h(x) \in k[x]\right\}$.

Lemma 4.4. Let $\alpha \in B \subset k$ and $j \in \mathbb{Z}$. Then

(i) $\varphi_{\beta}\left((x-\alpha)^{j}\right) \in \mathrm{B}^{1}(\mathcal{U}, \mathcal{O})$ for all $\beta \in B_{\infty}$,

(ii) $\varphi_{\alpha}\left(y(x-\alpha)^{j}\right) \in \mathrm{B}^{1}\left(\right.$ (U, O) if $j>c_{\alpha}$, and

(iii) $\varphi_{\infty}\left(y(x-\alpha)^{j}\right) \in \mathrm{B}^{1}(\mathcal{U}, 0)$ if $j \leq 0$.

Proof. (i) Suppose that $\beta \in B$. If $\beta \neq \alpha$ or if $j \geq 0$, then $(x-\alpha)^{j}$ is regular at $P_{\beta}$, and so $\varphi_{\beta}\left((x-\alpha)^{j}\right) \in \mathrm{B}^{1}(\mathcal{U}, \mathcal{O})$ by Lemma 4.1. For $j \geq 0$, it follows from this and Equation (4-2) that the cocycle $\varphi_{\infty}\left((x-\alpha)^{j}\right)=-\sum_{\beta \in B} \varphi_{\beta}\left((x-\alpha)^{j}\right)$ is a coboundary. If $j<0$, then $\varphi_{\infty}\left((x-\alpha)^{j}\right) \in \mathrm{B}^{1}(u, \mathcal{O})$ by Lemma 4.1.

Finally, $(x-\alpha)^{j} \in \Gamma\left(U_{\gamma}, \mathcal{O}\right)$ for all $\gamma \in B_{\infty}-\{\alpha\}$ if $\beta=\alpha \neq \infty$ and $j<0$. By Equation (4-2),

$$
\varphi_{\alpha}\left((x-\alpha)^{j}\right)=-\sum_{\gamma \in B_{\infty}-\{\alpha\}} \varphi_{\gamma}\left((x-\alpha)^{j}\right)=-\sum_{\gamma \in B_{\infty}-\{\alpha\}} \delta \psi_{\gamma}\left((x-\alpha)^{j}\right),
$$

which is a coboundary.

(ii) If $j>c_{\alpha}$, then $y(x-\alpha)^{j} \in \Gamma\left(U_{\alpha}, \mathbb{O}\right)$ and $\varphi_{\alpha}\left(y(x-\alpha)^{j}\right)=\delta \psi_{\alpha}\left(y(x-\alpha)^{j}\right)$.

(iii) If $j \leq 0$, then $y(x-\alpha)^{j} \in \Gamma\left(U_{\infty}, \mathcal{O}\right)$ and $\varphi_{\infty}\left(y(x-\alpha)^{j}\right)=\delta \psi_{\infty}\left(y(x-\alpha)^{j}\right)$. 
Consider the cocycles $\phi_{\alpha, j} \in \mathrm{Z}^{1}(U, \mathcal{O})$ for $\alpha \in B$ and $j \in \mathbb{Z}$ defined by

$$
\phi_{\alpha, j}:=\varphi_{\alpha}\left(y(x-\alpha)^{j}\right) .
$$

Given $\phi \in \mathrm{Z}^{1}(\mathcal{O}, \mathcal{O}), \tilde{\phi}$ denotes the cohomology class of $\phi$ in $\mathrm{H}^{1}(u, \mathcal{O})$. For $\alpha \in B_{\infty}$, define the map

$$
\tilde{\varphi}_{\alpha}: \Gamma\left(U^{\prime}, \mathcal{O}\right) \rightarrow \mathrm{H}^{1}(u, \mathcal{O}), \quad f \mapsto \varphi_{\alpha}(f) \bmod ^{1}(U, \mathcal{O}) .
$$

We now study $\mathrm{H}^{1}(u, \mathcal{O})$; the following lemma is a variant of a special case of [Madden 1978, Lemma 6]:

Lemma 4.5. A basis for $\mathrm{H}^{1}(U, O)$ is given by the cohomology classes $\tilde{\phi}_{\alpha, j}$ for $\alpha \in B$ and $1 \leq j \leq c_{\alpha}$ and $\tilde{\phi}_{\alpha, 0}$ for $\alpha \in B^{\prime}$.

Proof. The set of cohomology classes $S=\left\{\tilde{\phi}_{\alpha, j} \mid \alpha \in B, 1 \leq j \leq c_{\alpha}\right\} \cup\left\{\tilde{\phi}_{\alpha, 0} \mid \alpha \in B^{\prime}\right\}$ has cardinality $r+\sum_{\alpha \in B} c_{\alpha}=g$. By Lemmas 4.3(i) and 4.4(i), it suffices to show that $\varphi_{\beta}\left(y(x-\alpha)^{j}\right)$ is in the span of $S$ for $\alpha, \beta \in B$ and $j \in \mathbb{Z}$. By Lemmas 4.3(ii) and 4.4(ii), it suffices to show that the span of $S$ contains $\tilde{\phi}_{0,0}$ and $\tilde{\varphi}_{\beta}\left(y(x-\alpha)^{-j}\right)$ for $\alpha, \beta \in B$ and $j>0$.

The cocycle $\varphi_{\infty}(y)$ is a coboundary by Lemmas 4.1 and 4.2. Using this and Equation (4-2), one computes in $\mathrm{H}^{1}(U, \mathrm{O})$ that

$$
\tilde{\phi}_{0,0}=\tilde{\varphi}_{0}(y)+\tilde{\varphi}_{\infty}(y)=-\sum_{\beta \in B^{\prime}} \tilde{\varphi}_{\beta}(y)=-\sum_{\beta \in B^{\prime}} \tilde{\phi}_{\beta, 0},
$$

which is in the span of $S$.

Now consider $\tilde{\varphi}_{\beta}\left(y(x-\alpha)^{-j}\right)$ for $\alpha, \beta \in B$ and $j>0$. If $0=r:=\# B-1$, then this cocycle is a coboundary by Equation (4-2) and Lemma 4.4(iii).

Let $r>0$; first suppose that $\alpha \neq \beta$. Consider the rational function $h=(x-\alpha)^{-j}$, which has no pole at $\beta$. Write $h=T+E$, where $T$ is the degree- $c_{\beta}$ Taylor polynomial of $h$ at $\beta$. Then $\varphi_{\beta}(y h)=\varphi(y T)+\varphi(y E)$. Note that the function $E$ on $\mathbb{P}^{1}$ has a zero at $\beta$ of order at least $c_{\beta}+1$. Recall that $\operatorname{ord}_{P_{\beta}}(x-\beta)=2$, and observe that $\operatorname{ord}_{P_{\beta}}(E) \geq 2\left(c_{\beta}+1\right)=d_{\beta}+1$ on $X$. Since $\operatorname{ord}_{P_{\beta}}(y)=-d_{\beta}$, it follows that $y E \in \Gamma\left(U_{\beta}, \mathcal{O}\right)$ and thus $\varphi_{\beta}(y E) \in \mathrm{B}^{1}(u, \mathcal{O})$ by Lemma 4.1. The term $\varphi_{\beta}(y T)$ is, by construction, a linear combination of $\varphi_{\beta}\left(y(x-\beta)^{j}\right)=\phi_{\beta, j}$ for $0 \leq j \leq c_{\beta}$. Thus, $\tilde{\varphi}_{\beta}(y h)$ is in the span of $S$, which completes the case when $\alpha \neq \beta$.

If $\alpha=\beta$ and $j>0$, one can reduce to the previous case by adding the coboundary $\varphi_{\infty}\left(y(x-\alpha)^{-j}\right)$ to $\varphi_{\alpha}\left(y(x-\alpha)^{-j}\right)$ and using Equation (4-2) to see that

$$
\tilde{\varphi}_{\alpha}\left(y(x-\alpha)^{-j}\right)=-\sum_{\gamma \in B-\{\alpha\}} \tilde{\varphi}_{\gamma}\left(y(x-\alpha)^{-j}\right) .
$$

The next lemma is important for describing the $F$-module structure of $\mathrm{H}^{1}(U, \mathcal{O})$. 
Lemma 4.6. If $\alpha \in B$ and $j \geq 0$, then

$$
F \tilde{\phi}_{\alpha, j}= \begin{cases}\tilde{\phi}_{\alpha, 2 j} & \text { if } 2 j \leq c_{\alpha}, \\ 0 & \text { otherwise. }\end{cases}
$$

Proof. Since $\left(F \phi_{\alpha, j}\right)_{\beta \gamma}=\left(\phi_{\alpha, j}\right)_{\beta \gamma}^{2}$, one computes that

$$
\begin{aligned}
\left(y(x-\alpha)^{j}\right)^{2} & =(y+f(x))(x-\alpha)^{2 j} \\
& =y(x-\alpha)^{2 j}+f(x)(x-\alpha)^{2 j} .
\end{aligned}
$$

The statement follows from the definition of $\tilde{\phi}_{\alpha, j}$ and Lemma 4.4(i).

Now define

$$
\begin{aligned}
W_{\alpha, \mathrm{ss}}^{\prime \prime} & :=\left\langle\tilde{\phi}_{\alpha, 0}\right\rangle & & \text { for } \alpha \in B^{\prime} \text { and } \\
W_{\alpha, \text { nil }}^{\prime \prime} & :=\left\langle\tilde{\phi}_{\alpha, j} \mid 1 \leq j \leq c_{\alpha}\right\rangle & & \text { for } \alpha \in B .
\end{aligned}
$$

Lemma 4.7. The subspaces $W_{\alpha, \mathrm{ss}}^{\prime \prime}$ and $W_{\alpha, \text { nil }}^{\prime \prime}$ of $\mathrm{H}^{1}(\mathcal{U}, \mathcal{O})$ are stable under the action of Frobenius for each $\alpha \in B$. There is an isomorphism of $F$-modules

$$
\mathrm{H}^{1}(U, \mathcal{O}) \simeq \bigoplus_{\alpha \in B^{\prime}} W_{\alpha, \mathrm{ss}}^{\prime \prime} \oplus \bigoplus_{\alpha \in B} W_{\alpha, \mathrm{nil}}^{\prime \prime} .
$$

Proof. This follows immediately from Lemmas 4.5 and 4.6.

4d. Auxiliary map. The next goal is to define a section $\sigma: \mathrm{H}^{1}(X, O) \rightarrow \mathrm{H}_{\mathrm{dR}}^{1}(X)$. To do this, the first step will be to define a homomorphism $\rho: Z^{1}(U, \mathcal{O}) \rightarrow \mathrm{C}^{0}\left(U, \Omega^{1}\right)$ by defining its components $\rho_{\alpha}: Z^{1}(U, \mathcal{O}) \rightarrow \Gamma\left(U_{\beta}, \Omega^{1}\right)$ for $\alpha, \beta \in B$. Given $\phi \in \mathrm{Z}^{1}(U, \mathcal{O})$ and $\alpha \in B$, the idea is to separate $d \phi$ into two parts: The first part will be regular at $P_{\alpha}$ and thus belong to $\Gamma\left(U_{\alpha}, \Omega^{1}\right)$, and the second part will be regular away from $P_{\alpha}$ and hence belong to $\Gamma\left(U_{\beta}, \Omega^{1}\right)$ for every $\beta \neq \alpha$.

Notation 4.8. Define the truncation operator $\Theta_{\geq i}: k\left[x, x^{-1}\right] \rightarrow k\left[x, x^{-1}\right]$ by

$$
\Theta_{\geq i}\left(\sum_{j} a_{j} x^{j}\right):=\sum_{j \geq i} a_{j} x^{j} .
$$

Operators $\Theta_{>i}, \Theta_{\leq i}, \Theta_{<i}: k\left[x, x^{-1}\right] \rightarrow k\left[x, x^{-1}\right]$ can be defined analogously. These operators can also be defined on $k\left[x_{\alpha}, x_{\alpha}^{-1}\right]$. To clarify some ambiguity in notation, if $m\left(x_{\alpha}\right) \in k\left[x_{\alpha}, x_{\alpha}^{-1}\right]$, then let $\Theta_{\geq i}\left(m\left(x_{\alpha}\right)\right)$ denote $\left.\Theta_{\geq i}(m(x))\right|_{x=x_{\alpha}}$.

Recall that $x_{\alpha}:=(x-\alpha)^{-1}$, and so $\phi_{\alpha, j}=\varphi_{\alpha}\left(y x_{\alpha}^{-j}\right)$. Then

$$
d\left(y x_{\alpha}^{-j}\right)=-j x_{\alpha}^{-j-1} y d x_{\alpha}+x_{\alpha}^{-j} d y .
$$


Using partial fractions and the fact that $d y=-d(f(x))$, one sees that

$$
d y=-\sum_{\beta \in B} f_{\beta}^{\prime}\left(x_{\beta}\right) d x_{\beta} .
$$

In light of these facts, consider the following definition:

Notation 4.9. For $\alpha \in B$ and $j \geq 0$, define

$$
R_{\alpha, j}:=\Theta_{\geq 0}\left(x_{\alpha}^{-j} f_{\alpha}^{\prime}\left(x_{\alpha}\right)\right) d x_{\alpha} \quad \text { and } \quad S_{\alpha, j}:=d\left(y x_{\alpha}^{-j}\right)+R_{\alpha, j} .
$$

Remark 4.10. Let $a_{\alpha, i} \in k$ be the coefficients of the (odd-power) monomials of the polynomials $f_{\alpha}\left(x_{\alpha}\right)$ defined in the partial fraction decomposition (1-1):

$$
f_{\alpha}\left(x_{\alpha}\right)=\sum_{i=0}^{c_{\alpha}} a_{\alpha, i} x_{\alpha}^{2 i+1} .
$$

Then

$$
R_{\alpha, j}=\sum_{j / 2 \leq i \leq c_{\alpha}} a_{\alpha, i} x_{\alpha}^{2 i-j} d x_{\alpha}=\sum_{j / 2 \leq i \leq c_{\alpha}} a_{\alpha, i} \omega_{\alpha, 2 i-j+1} .
$$

Lemma 4.11. Let $\alpha \in B$ and $j \geq 0$.

(1) The differential form $R_{\alpha, j}$ is regular away from $P_{\alpha}$, that is, $R_{\alpha, j} \in \Gamma\left(U_{\beta}, \Omega^{1}\right)$ for all $\beta \in B_{\infty}-\{\alpha\}$

(2) The differential form $S_{\alpha, j}$ is regular at $P_{\alpha}$ for $0 \leq j \leq c_{\alpha}$, that is, $S_{\alpha, j} \in$ $\Gamma\left(U_{\alpha}, \Omega^{1}\right)$.

Proof. (1) This follows from Remark 4.10 and Equation (3-2).

(2) By Notations 4.8 and 4.9 and Equations (4-5) and (4-6), one sees that

$$
\begin{aligned}
S_{\alpha, j} & =d\left(y x_{\alpha}^{-j}\right)+\Theta_{\geq 0}\left(x_{\alpha}^{-j} f_{\alpha}^{\prime}\left(x_{\alpha}\right)\right) d x_{\alpha} \\
& =-j x_{\alpha}^{-j-1} y d x_{\alpha}-\Theta_{<0}\left(x_{\alpha}^{-j} f_{\alpha}^{\prime}\left(x_{\alpha}\right)\right) d x_{\alpha}-\sum_{\beta \in B-\{\alpha\}} x_{\alpha}^{-j} f_{\beta}^{\prime}\left(x_{\beta}\right) d x_{\beta} .
\end{aligned}
$$

In the first part of Equation (4-8), note that the order of vanishing of $x_{\alpha}^{-j-1} y d x_{\alpha}$ at $P_{\alpha}$ is $2 d_{\alpha}-1+2 j$ by Lemma 4.2 and Equation (3-1), so this term is regular at $P_{\alpha}$.

In the second part of Equation (4-8), note that $\Theta_{<0}\left(x_{\alpha}^{-j} f_{\alpha}^{\prime}\left(x_{\alpha}\right)\right)$ is contained in $x_{\alpha}^{-1} k\left[x_{\alpha}^{-1}\right]$. Thus, $\Theta_{<0}\left(x_{\alpha}^{-j} f_{\alpha}^{\prime}\left(x_{\alpha}\right)\right)$ has a zero of order at least 2 at $P_{\alpha}$. As seen in the proof of Lemma 3.1, $d x_{\alpha}$ has a zero of order $d_{\alpha}-3$ at $P_{\alpha}$. Thus, $\Theta_{<0}\left(x_{\alpha}^{-j} f_{\alpha}^{\prime}\left(x_{\alpha}\right)\right) d x_{\alpha}$ is regular at $P_{\alpha}$.

The last part of Equation (4-8) is regular at $P_{\alpha}$ since $x_{\alpha}^{-1}$ and $f_{\beta}^{\prime}\left(x_{\beta}\right) d x_{\beta}$ are regular at $P_{\alpha}$.

4e. Definition of $\rho$. We define a $k$-linear morphism

$$
\rho: \mathrm{Z}^{1}(u, \mathcal{O}) \rightarrow \mathrm{C}^{0}\left(u, \Omega^{1}\right) \text {. }
$$


4e.1. Definition of $\rho$ on $\mathrm{B}^{1}(\mathcal{(}, \mathcal{O})$. If $\phi \in \mathrm{B}^{1}(u, \mathcal{O})$, then for some $\kappa \in \mathrm{C}^{0}(u, \mathcal{O})$, $\phi=\delta \kappa$. Define

$$
\rho(\phi):=d \kappa
$$

with differentiation performed component-wise. This map is well-defined since if $\kappa$ is regular at $P \in X(k)$, then so is $d \kappa$. Moreover, if $\kappa^{\prime}$ is another element such that $\phi=\delta \kappa^{\prime}$, then $\delta\left(\kappa-\kappa^{\prime}\right)=0$, and therefore, $\kappa-\kappa^{\prime} \in \mathrm{H}^{0}(u, \mathcal{O})$ is constant and annihilated by $d$. Let $\rho_{\beta}(\phi)$ denote $(\rho(\phi))_{\beta}$.

It follows from the definition that $\mathscr{C}\left(\rho\left(\mathrm{B}^{1}(\mathcal{U}, \mathcal{O})\right)\right)=0$ since the Cartier operator annihilates all exact differential forms. Explicitly, the map $\rho$ is computed as follows:

Lemma 4.12. (i) If $\alpha \in B_{\infty}$ and $h \in \Gamma\left(U_{\alpha}, \mathcal{O}\right)$, then $\rho \varphi_{\alpha}\left(\left.h\right|_{U^{\prime}}\right)=d \psi_{\alpha}(h)$.

(ii) If $\alpha \in B$ and $j \leq 0$, then

$$
\rho \varphi_{\alpha}\left((x-\alpha)^{j}\right)=-\sum_{\gamma \in B_{\infty}-\{\alpha\}} d \psi_{\gamma}\left((x-\alpha)^{j}\right) .
$$

Proof. (i) This is immediate from the definition of the map $\rho$ and Lemma 4.1.

(ii) This follows from part (i), Equation (4-4), and the definition of $\rho$.

Example 4.13. We find the value of $\rho$ on the 1-coboundary $\varphi_{\alpha}\left(f(x) x_{\alpha}^{-j}\right)$ if $\alpha \in B$ and $j \geq 0$. Let

$$
r_{\alpha, j}:=\Theta_{>0}\left(x_{\alpha}^{-j} f_{\alpha}\left(x_{\alpha}\right)\right) \quad \text { and } \quad s_{\alpha, j}:=\Theta_{\leq 0}\left(x_{\alpha}^{-j} f_{\alpha}\left(x_{\alpha}\right)\right)+\sum_{\beta \neq \alpha} x_{\alpha}^{-j} f_{\beta}\left(x_{\beta}\right) .
$$

Then

$$
f(x) x_{\alpha}^{-j}=r_{\alpha, j}+s_{\alpha, j},
$$

and $r_{\alpha, j}$ has a pole at $P_{\alpha}$ but is regular everywhere else while $s_{\alpha, j}$ is regular at $P_{\alpha}$, so

$$
\varphi_{\alpha}\left(f(x) x_{\alpha}^{-j}\right)=\delta \psi_{\alpha}\left(s_{\alpha, j}\right)-\sum_{\beta \in B_{\infty}-\{\alpha\}} \delta \psi_{\beta}\left(r_{\alpha, j}\right) .
$$

Therefore, for $\beta \neq \alpha$, by Lemma 4.12, $\rho_{\beta} \varphi_{\alpha}\left(f(x) x_{\alpha}^{-j}\right)=-d\left(r_{\alpha, j}\right)$. Since $f_{\alpha}\left(x_{\alpha}\right) \in x_{\alpha} k\left[x_{\alpha}^{2}\right]$, this simplifies to

$$
\rho_{\beta} \varphi_{\alpha}\left(f(x) x_{\alpha}^{-j}\right)= \begin{cases}-R_{\alpha, j} & \text { if } j \text { is even, } \\ 0 & \text { if } j \text { is odd }\end{cases}
$$

Similarly,

$$
\rho_{\alpha} \varphi_{\alpha}\left(f(x) x_{\alpha}^{-j}\right)= \begin{cases}-S_{\alpha, j} & \text { if } j \text { is even } \\ d\left(f(x) x_{\alpha}^{-j}\right) & \text { if } j \text { is odd. }\end{cases}
$$


4e.2. Definition of $\rho_{\beta}$ on $\mathrm{Z}^{1}(u, \mathcal{O})$. By Lemma $4.5, \mathrm{Z}^{1}(\mathcal{U}, \mathcal{O})$ is generated by $\mathrm{B}^{1}(U, \mathcal{O})$ and $\phi_{\alpha, j}$ for $\alpha \in B$ and $0 \leq j \leq c_{\alpha}$. For $\alpha, \beta \in B$, define

$$
\rho_{\beta}\left(\phi_{\alpha, j}\right):= \begin{cases}R_{\alpha, j} & \text { if } \beta \neq \alpha, \\ S_{\alpha, j} & \text { if } \beta=\alpha,\end{cases}
$$

and extend $\rho_{\beta}$ to $Z^{1}(U, \mathcal{O})$ linearly. For all $\beta \in B-\{\alpha\}$, note that

$$
\rho_{\alpha}\left(\phi_{\alpha, j}\right)=d\left(y x_{\alpha}^{-j}\right)+\rho_{\beta}\left(\phi_{\alpha, j}\right) .
$$

Lemma 4.14. There is a well-defined map $\rho: Z^{1}(U, \mathcal{O}) \rightarrow C^{0}\left(U, \Omega^{1}\right)$ given by

$$
\rho:=\bigoplus_{\beta \in B_{\infty}} \rho_{\beta} .
$$

Proof. By Section 4e.1 and Lemma 4.11, $\rho_{\beta}\left(\mathrm{Z}^{1}(\mathcal{U}, \mathcal{O})\right) \subset \Gamma\left(U_{\beta}, \Omega^{1}\right)$ if $\beta \in B_{\infty}$.

Here is an example of a computation of the map $\rho$.

Lemma 4.15. Let $\alpha \in B$ and $j \geq 0$. For each $\beta \in B$, in $\Gamma\left(U_{\beta}, \Omega^{1}\right)$,

$$
\rho_{\beta} \varphi_{\alpha}\left(y^{2} x_{\alpha}^{-2 j}\right)= \begin{cases}0 & \text { if } 0 \leq 2 j \leq c_{\alpha}, \\ -R_{\alpha, 2 j} & \text { if } 2 j>c_{\alpha} .\end{cases}
$$

In particular, $\rho \varphi_{\alpha}\left(y^{2} x_{\alpha}^{-2 j}\right)$ lies in the subspace $W_{\alpha, \text { nil }}^{\prime}$ of $\mathrm{H}^{0}\left(U, \Omega^{1}\right)$.

Proof. We have $y^{2} x_{\alpha}^{-2 j}=y x_{\alpha}^{-2 j}+f(x) x_{\alpha}^{-2 j}$, and therefore

$$
\varphi_{\alpha}\left(y^{2} x_{\alpha}^{-2 j}\right)=\phi_{\alpha, 2 j}+\varphi_{\alpha}\left(f(x) x_{\alpha}^{-2 j}\right) .
$$

Suppose $0 \leq 2 j \leq c_{\alpha}$. If $\beta \neq \alpha$, then $\rho_{\beta}\left(\phi_{\alpha, 2 j}\right)=R_{\alpha, 2 j}=-\rho_{\beta}\left(\varphi_{\alpha}\left(f(x) x_{\alpha}^{-2 j}\right)\right)$ by Equation (4-9). By Equation (4-10), $\rho_{\alpha}\left(\phi_{\alpha, 2 j}\right)=S_{\alpha, 2 j}=-\rho_{\alpha}\left(\varphi_{\alpha}\left(f(x) x_{\alpha}^{-2 j}\right)\right)$. Thus, $\rho\left(\phi_{\alpha, 2 j}\right)+\rho\left(\varphi_{\alpha}\left(f(x) x_{\alpha}^{-2 j}\right)\right)=0$.

Now, suppose that $2 j>c_{\alpha}$. Then $y x_{\alpha}^{-2 j}$ is regular at $P_{\alpha}$, and therefore, $\phi_{\alpha, 2 j}$ is a coboundary with $\rho\left(\phi_{\alpha, 2 j}\right)=d \varphi_{\alpha}\left(y x_{\alpha}^{2 j}\right)$. Therefore, for $\beta \neq \alpha$,

$$
\rho_{\beta}\left(\phi_{\alpha, 2 j}\right)+\rho_{\beta}\left(\varphi_{\alpha}\left(f(x) x_{\alpha}^{-2 j}\right)\right)=-R_{\alpha, 2 j},
$$

and

$$
\rho_{\alpha}\left(\phi_{\alpha, 2 j}\right)+\rho_{\alpha}\left(\varphi_{\alpha}\left(f(x) x_{\alpha}^{-2 j}\right)\right)=d\left(y x_{\alpha}^{-2 j}\right)+d\left(f(x) x_{\alpha}^{-2 j}\right)-R_{\alpha, 2 j}=-R_{\alpha, 2 j} .
$$

By Remark 4.10, $R_{\alpha, 2 j} \in\left\langle\omega_{\alpha, 2 i-2 j+1} \mid j \leq i \leq c_{\alpha}\right\rangle$. If $2 j>c_{\alpha}$ and $j \leq i \leq c_{\alpha}$, then $1 \leq 2 i-2 j+1 \leq c_{\alpha}$, and so $R_{\alpha, 2 j} \in W_{\alpha, \text { nil }}^{\prime}$. Finally, since $\rho_{\beta} \varphi_{\alpha}\left(y^{2} x_{\alpha}^{-2 j}\right)$ is independent of the choice of $\beta \in B_{\infty}$, we have $\rho \varphi_{\alpha}\left(y^{2} x_{\alpha}^{-2 j}\right)$ lies in the kernel $\mathrm{H}^{0}\left(u, \Omega^{1}\right)$ of the coboundary map $\delta: C^{0}\left(u, \Omega^{1}\right) \rightarrow C^{1}\left(u, \Omega^{1}\right)$.

Lemma 4.16. (i) If $\phi \in \mathrm{Z}^{1}(\mathcal{U}, \mathcal{O})$, then $\delta \rho(\phi)=d \phi$.

(ii) In particular, $\mathscr{C}\left(\rho_{\alpha}(\phi)\right)=\mathscr{C}\left(\rho_{\beta}(\phi)\right)$ for all $\alpha, \beta \in B_{\infty}$. 
(iii) For all $\alpha \in B$ and $\beta \in B_{\infty}$, we have $\mathscr{C}\left(\rho_{\beta}\left(\phi_{\alpha, j}\right)\right)=\mathscr{C}\left(R_{\alpha, j}\right)$.

Proof. (i) The definition of $\rho_{\beta}$ implies that $\rho_{\alpha}(\phi)-\rho_{\beta}(\phi)=d(\phi)_{\alpha \beta}$ for all $\alpha, \beta \in B_{\infty}$.

(ii) This follows from part (i) since the Cartier operator annihilates exact differential forms.

(iii) This follows from part (ii) and the definition of $\rho_{\beta}$.

Remark 4.17. With $a_{\alpha, i}$ defined as in Remark 4.10, one can explicitly compute

$$
\mathscr{C}\left(R_{\alpha, j}\right)= \begin{cases}\sum_{i=(j+1) / 2}^{c_{\alpha}} \sqrt{a_{\alpha, i}} \omega_{\alpha, i-(j-1) / 2} & \text { if } j \text { is odd, } \\ 0 & \text { if } j \text { is even. }\end{cases}
$$

In particular, $\mathscr{C}\left(R_{\alpha, j}\right) \in W_{\alpha, \text { nil }}^{\prime}$.

4f. The $\mathbb{E}$-module structure of the de Rham cohomology. Consider the exact sequence of $\mathbb{E}$-modules

$$
0 \rightarrow \mathrm{H}^{0}\left(X, \Omega^{1}\right) \stackrel{\lambda}{\rightarrow} \mathrm{H}_{\mathrm{dR}}^{1}(X) \stackrel{\gamma}{\rightarrow} \mathrm{H}^{1}(X, \mathcal{O}) \rightarrow 0,
$$

where $\mathbb{E}=k[F, V]$ is the noncommutative ring defined in Section 2a. Consider the $k$-linear function

$$
\sigma: \mathrm{H}^{1}(X, \mathcal{O}) \rightarrow \mathrm{H}_{\mathrm{dR}}^{1}(X)
$$

defined by $\sigma(\phi)=(\phi, \rho(\phi))$ for $\phi \in \mathrm{Z}^{1}(U, \mathcal{O})$.

Lemma 4.18. The function $\sigma$ is a section of $\gamma: \mathrm{H}_{\mathrm{dR}}^{1}(X) \rightarrow \mathrm{H}^{1}(X, 0)$.

Proof. The function $\sigma$ is well-defined because $\sigma\left(\mathrm{B}^{1}(U, O)\right) \subset \mathrm{B}_{\mathrm{dR}}^{1}(U)$ by the definition of $\rho_{\beta}$ on $\mathrm{B}^{1}(\mathcal{O}, \mathcal{O})$. It is clearly a section of $\gamma$.

Note that $\sigma$ is not a splitting of $\mathbb{E}$-modules.

For $\alpha \in B$, let $\lambda_{\alpha, j}:=\lambda\left(\omega_{\alpha, j}\right)$ and $\sigma_{\alpha, j}:=\sigma\left(\tilde{\phi}_{\alpha, j}\right)$.

Proposition 4.19. For $0 \leq j \leq c_{\alpha}$, the action of $F$ and $V$ on $\mathrm{H}_{\mathrm{dR}}^{1}(X)$ is given by

(i) $F \lambda_{\alpha, j}=0$,

(ii) $V \lambda_{\alpha, j}= \begin{cases}\lambda_{\alpha, j / 2} & \text { if } j \text { is even, } \\ 0 & \text { if } j \text { is odd, }\end{cases}$

(iii) $F \sigma_{\alpha, j}= \begin{cases}\sigma_{\alpha, 2 j} & \text { if } j \leq c_{\alpha} / 2 \\ \lambda\left(R_{\alpha, 2 j}\right) & \text { if } j>c_{\alpha} / 2\end{cases}$

(iv) $V \sigma_{\alpha, j}= \begin{cases}\lambda\left(\mathscr{C}\left(R_{\alpha, j}\right)\right) & \text { if } j \text { is odd, } \\ 0 & \text { if } j \text { is even. }\end{cases}$

Proof. (i) This follows from Section 2f.

(ii) This follows from Lemma 3.2 after applying $\lambda$. 
(iii) In $\mathrm{Z}_{\mathrm{dR}}^{1}(U)$,

$$
\begin{aligned}
F\left(\sigma_{\alpha, j}\right) & =\left(F \phi_{\alpha, j}, 0\right) \\
& =\left(\varphi_{\alpha}\left(y^{2} x_{\alpha}^{-2 j}\right), \rho \varphi_{\alpha}\left(y^{2} x_{\alpha}^{-2 j}\right)\right)-\left(0, \rho \varphi_{\alpha}\left(y^{2} x_{\alpha}^{-2 j}\right)\right) \\
& =\sigma \varphi_{\alpha}\left(y^{2} x_{\alpha}^{-2 j}\right)-\left(0, \rho \varphi_{\alpha}\left(y^{2} x_{\alpha}^{-2 j}\right)\right) .
\end{aligned}
$$

Since $y^{2} x_{\alpha}^{-2 j}=y x_{\alpha}^{-2 j}+f(x) x_{\alpha}^{-2 j}$, linearity of $\sigma$ and $\varphi_{\alpha}$ yields that

$$
\sigma \varphi_{\alpha}\left(y^{2} x_{\alpha}^{-2 j}\right)=\sigma \varphi_{\alpha}\left(y x_{\alpha}^{-2 j}\right)+\sigma \varphi_{\alpha}\left(f(x) x_{\alpha}^{-2 j}\right) .
$$

The term $\sigma \varphi_{\alpha}\left(f(x) x_{\alpha}^{-2 j}\right)$ is a coboundary by Lemma 4.4(i), and $\sigma \varphi_{\alpha}\left(y x_{\alpha}^{-2 j}\right)$ equals $\sigma_{\alpha, 2 j}$ if $0 \leq 2 j \leq c_{\alpha}$ and is a coboundary if $2 j>c_{\alpha}$ by Lemma 4.4(ii). By Lemma 4.15,

$$
\left(0, \rho \varphi_{\alpha}\left(y^{2} x_{\alpha}^{-2 j}\right)\right)= \begin{cases}0 & \text { if } 0 \leq 2 j \leq c_{\alpha}, \\ -\lambda\left(R_{\alpha, 2 j}\right) & \text { if } 2 j>c_{\alpha} .\end{cases}
$$

(iv) Since $V(\phi, \rho(\phi))=(0, \mathscr{C}(\rho(\phi)))$, the result follows by Lemma 4.16(iii).

Consider the subspaces of $\mathrm{H}_{\mathrm{dR}}^{1}(X)$ given by

$$
\begin{aligned}
W_{\alpha, \text { ss }} & :=\left\langle\lambda_{\alpha, 0}-\lambda_{0,0}, \sigma_{\alpha, 0}\right\rangle, \\
W_{\alpha, \text { nil }} & :=\left\langle\lambda_{\alpha, j}, \sigma_{\alpha, j} \mid 1 \leq j \leq c_{\alpha}\right\rangle .
\end{aligned}
$$

Theorem 4.20. The subspaces $W_{\alpha, \mathrm{ss}}$ and $W_{\alpha, \text { nil }}$ of $\mathrm{H}_{\mathrm{dR}}^{1}(X)$ are stable under the action of Frobenius and Verschiebung for each $\alpha \in B$. There is an isomorphism of $\mathbb{E}$-modules

$$
\mathrm{H}_{\mathrm{dR}}^{1}(X)=\bigoplus_{\alpha \in B^{\prime}} W_{\alpha, \mathrm{ss}} \oplus \bigoplus_{\alpha \in B} W_{\alpha, \text { nil }}
$$

Proof. The stability is immediate by Proposition 4.19, Remark 4.10, and Lemma 4.15. The decomposition follows from Lemmas 4.18, 3.3, and 4.7.

Theorem 1.2 is immediate from Theorem 4.20.

\section{Results on the Ekedahl-Oort type}

For a natural number $c$, let $G_{c}$ be the unique symmetric $\mathrm{BT}_{1}$ group scheme of rank $p^{2 c}$ with Ekedahl-Oort type $[0,1,1,2,2, \ldots,\lfloor c / 2\rfloor]$. In other words, this means that there is a final filtration $N_{1} \subset N_{2} \subset \cdots \subset N_{2 c}$ of $D\left(G_{c}\right)$ as a $k$-vector space, which is stable under the action of $V$ and $F^{-1}$ and with $i=\operatorname{dim}\left(N_{i}\right)$, such that $\operatorname{dim}\left(V\left(N_{i}\right)\right)=\lfloor i / 2\rfloor$. In Section 5a, we prove that group schemes of the form $G_{c}$ appear in the decomposition of $J_{X}$ [2] when $X$ is a hyperelliptic $k$-curve. In Section 5b, we describe the Dieudonné module of $G_{c}$ for arbitrary $c$ and give examples. 
5a. The final filtration for hyperelliptic curves in characteristic 2. Suppose $X$ is a hyperelliptic $k$-curve with affine equation $y^{2}-y=f(x)$ as described in Notation 1.1. For $\alpha \in B$, recall that $c_{\alpha}=\left(d_{\alpha}-1\right) / 2$, where $d_{\alpha}$ is the ramification invariant of $X$ above $\alpha$. Recall the subspaces $W_{\alpha \text {,nil }}$ of $\mathrm{H}_{\mathrm{dR}}^{1}(X)$ from Section $4 \mathrm{f}$. Define subspaces $N_{\alpha, i}$ of $W_{\alpha, \text { nil }}$ for $0 \leq i \leq 2 c_{\alpha}$ as follows: $N_{\alpha, 0}:=\{0\}$ and

$$
N_{\alpha, i}:= \begin{cases}\left\langle\lambda_{\alpha, j} \mid 1 \leq j \leq i\right\rangle & \text { if } 1 \leq i \leq c_{\alpha}, \\ N_{\alpha, c_{\alpha}} \oplus\left\langle\sigma_{\alpha, j} \mid 1 \leq j \leq i\right\rangle & \text { if } c_{\alpha}+1 \leq i \leq 2 c_{\alpha} .\end{cases}
$$

Proposition 5.1. The filtration $N_{\alpha, 0} \subset N_{\alpha, 1} \subset N_{\alpha, 2} \subset \cdots \subset N_{\alpha, 2 c_{\alpha}}$ is a final filtration of $W_{\alpha, \text { nil }}$ for each $\alpha \in B$. Furthermore, $V\left(N_{\alpha, i}\right)=N_{\alpha,\lfloor i / 2\rfloor}$.

Proof. Let $0 \leq i \leq 2 c_{\alpha}$. Then $\operatorname{dim}\left(N_{\alpha, i}\right)=i$. By Proposition 4.19, $V\left(N_{\alpha, i}\right)=N_{\alpha,\lfloor i / 2\rfloor}$, and $F^{-1}\left(N_{\alpha, i}\right)=N_{\alpha, c_{\alpha}+\lceil i / 2\rceil}$. Thus, the filtration $N_{\alpha, 0} \subset N_{\alpha, 1} \subset N_{\alpha, 2} \subset \cdots \subset N_{\alpha, 2 c_{\alpha}}$ is stable under the action of $V$ and $F^{-1}$.

Theorem 5.2. Let $k$ be an algebraically closed field of characteristic $p=2$. Let $X$ be a hyperelliptic $k$-curve with affine equation $y^{2}-y=f(x)$ as described in Notation 1.1. Then the 2-torsion group scheme of $X$ decomposes as

$$
J_{X}[2] \simeq\left(\mathbb{Z} / 2 \oplus \mu_{2}\right)^{r} \oplus \bigoplus_{\alpha \in B} G_{c_{\alpha}},
$$

and the a-number of $X$ is

$$
a_{X}=\left(g+1-\#\left\{\alpha \in B \mid d_{\alpha} \equiv 1 \bmod 4\right\}\right) / 2 .
$$

Proof. By [Oda 1969, Section 5], there is an isomorphism of $\mathbb{E}$-modules between the Dieudonné module $D\left(J_{X}[2]\right)$ and the de Rham cohomology $\mathrm{H}_{\mathrm{dR}}^{1}(X)$. By Theorem 4.20, there is an isomorphism of $\mathbb{E}$-modules

$$
\mathrm{H}_{\mathrm{dR}}^{1}(X)=\bigoplus_{\alpha \in B^{\prime}} W_{\alpha, \mathrm{ss}} \oplus \bigoplus_{\alpha \in B} W_{\alpha, \text { nil }}
$$

If $\alpha \in B^{\prime}$, then $W_{\alpha, \mathrm{ss}}$ is isomorphic to $\mathbb{E} / \mathbb{E}(F, 1-V) \oplus \mathbb{E} / \mathbb{E}(V, 1-F) \simeq D\left(\mathbb{Z} / 2 \oplus \mu_{2}\right)$. Finally, Proposition 5.1 shows $W_{\alpha \text {,nil }} \simeq D\left(G_{c_{\alpha}}\right)$, which completes the proof of the statement about $J_{X}[2]$. The statement about $a_{X}$ can be found in Proposition 3.4.

As a corollary, we highlight the special case when $r=0$ (for example, when $f(x) \in k[x])$. Corollary 5.3 is stated without proof in [van der Geer 1999, 3.2].

Corollary 5.3. Let $k$ be an algebraically closed field of characteristic $p=2$. Suppose $X$ is a hyperelliptic $k$-curve of genus $g$ and p-rank $r=0$. Then the EkedahlOort type of $J_{X}[2]$ is $[0,1,1,2,2, \ldots,\lfloor g / 2\rfloor]$, and the -number $a_{X}=\lfloor(g+1) / 2\rfloor$.

Proof. This is a special case of Theorem 5.2 where $\# B=1$. 
The next immediate corollary of Theorem 5.2 is included to emphasize that Theorem 5.2 gives a complete classification of the 2-torsion group schemes that occur as $J_{X}[2]$ when $X$ is a hyperelliptic $k$-curve.

Corollary 5.4. Let $k$ be an algebraically closed field of characteristic $p=2$. Let $G$ be a polarized $\mathrm{BT}_{1}$ group scheme over $k$ of rank $p^{2 g}$. Let $0 \leq r \leq g$. Then $G \simeq J_{X}$ [2] for some hyperelliptic $k$-curve $X$ of genus $g$ and p-rank $r$ if and only if there exist nonnegative integers $c_{1}, \ldots, c_{r+1}$ such that $\sum_{i=1}^{r+1} c_{i}=g-r$ and such that

$$
G \simeq\left(\mathbb{Z} / 2 \oplus \mu_{2}\right)^{r} \oplus \bigoplus_{\alpha \in B} G_{c_{\alpha}} .
$$

Remark 5.5. For fixed $g$, the number of isomorphism classes of polarized $\mathrm{BT}_{1}$ group schemes of rank $p^{2 g}$ that occur as $J_{X}$ [2] for some hyperelliptic $k$-curve $X$ of genus $g$ equals the number of partitions of $g+1$. To see this, note that the isomorphism class of $J_{X}[2]$ is determined by the multiset $\left\{d_{1}, \ldots, d_{r+1}\right\}$, where $d_{i}=2 c_{i}+1$ and $\sum_{i=1}^{r+1}\left(d_{i}+1\right)=2 g+2$. So the number of isomorphism classes equals the number of partitions of $2 g+2$ into positive even integers.

Remark 5.6. The examples in Section 5 b show that the factors $G_{c}$ appearing in the decomposition of $J_{X}$ [2] in Theorem 5.2 may not be indecomposable as polarized $\mathrm{BT}_{1}$ group schemes.

5b. Description of a particular Ekedahl-Oort type. Recall that $G_{c}$ is the unique polarized $\mathrm{BT}_{1}$ group scheme over $k$ of rank $p^{2 c}$ that has Ekedahl-Oort type $[0,1,1,2,2, \ldots,\lfloor c / 2\rfloor]$. Recall that $\mathbb{E}=k[F, V]$ is the noncommutative ring defined in Section 2b. In this section, we describe the Dieudonné module $D\left(G_{c}\right)$. We start with some examples to motivate the notation. These show that $G_{c}$ is sometimes indecomposable and sometimes decomposes into polarized $\mathrm{BT}_{1}$ group schemes of smaller rank. The first four examples were found using preexisting tables.

Example 5.7. (i) For $c=1$, the Ekedahl-Oort type is [0]. This Ekedahl-Oort type occurs for the $p$-torsion group scheme of a supersingular elliptic curve. See [Goren 2002, Example A.3.14; Pries 2008, Example 2.3] for a description of $G_{1}$. It has Dieudonné module $\mathbb{E} / \mathbb{E}(F+V)$.

(ii) For $c=2$, the Ekedahl-Oort type is $[0,1]$. This Ekedahl-Oort type occurs for the $p$-torsion group scheme of a supersingular abelian surface that is not superspecial. See [Goren 2002, Example A.3.15; Pries 2008, Example 2.3] for a description of $G_{2}$. It has Dieudonné module $\mathbb{E} / \mathbb{E}\left(F^{2}+V^{2}\right)$.

(iii) For $c=3$, the Ekedahl-Oort type is $[0,1,1]$. This Ekedahl-Oort type occurs for an abelian threefold with $p$-rank 0 and $a$-number 2 whose $p$-torsion is indecomposable as a polarized $\mathrm{BT}_{1}$ group scheme. By [Pries 2008, Lemma 3.4], $G_{3}$ has Dieudonné module $\mathbb{E} / \mathbb{E}\left(F^{2}+V\right) \oplus \mathbb{E} / \mathbb{E}\left(V^{2}+F\right)$. 
(iv) For $c=4$, the Ekedahl-Oort type is $[0,1,1,2]$. This Ekedahl-Oort type occurs for an abelian fourfold with $p$-rank 0 and $a$-number 2 whose $p$-torsion decomposes as a direct sum of polarized $\mathrm{BT}_{1}$ group schemes of rank $p^{2}$ and $p^{6}$. By [Pries 2008, Table 4.4], $G_{4}$ has Dieudonné module $\mathbb{E} / \mathbb{E}(F+V) \oplus \mathbb{E} / \mathbb{E}\left(F^{3}+V^{3}\right)$.

We now provide an algorithm to determine the Dieudonné module $D\left(G_{c}\right)$ for all positive integers $c \in \mathbb{N}$ following the method of [Oort 2001, Section 9.1].

Proposition 5.8. The Dieudonné module $D\left(G_{c}\right)$ is the $\mathbb{E}$-module generated as a $k$-vector space by $\left\{X_{1}, \ldots, X_{c}, Y_{1}, \ldots, Y_{c}\right\}$ with the actions of $F$ and $V$ given by

(i) $F\left(Y_{j}\right)=0$,

(ii) $V\left(Y_{j}\right)= \begin{cases}Y_{2 j} & \text { if } j \leq c / 2, \\ 0 & \text { if } j>c / 2,\end{cases}$

(iii) $F\left(X_{i}\right)= \begin{cases}X_{j / 2} & \text { if } j \text { is even, } \\ Y_{c-(j-1) / 2} & \text { if } j \text { is odd }\end{cases}$

(iv) $V\left(X_{j}\right)= \begin{cases}0 & \text { if } j \leq(c-1) / 2, \\ -Y_{2 c-2 j+1} & \text { if } j>(c-1) / 2 .\end{cases}$

Proof. By definition of $G_{c}$, there is a final filtration $N_{1} \subset N_{2} \subset \cdots \subset N_{2 c}$ of $D\left(G_{c}\right)$ as a $k$-vector space, which is stable under the action of $V$ and $F^{-1}$ and with $i=\operatorname{dim}\left(N_{i}\right)$, such that $v_{i}:=\operatorname{dim}\left(V\left(N_{i}\right)\right)=\lfloor i / 2\rfloor$. This implies that $v_{i}=v_{i-1}$ if and only if $i$ is odd. In the notation of [Oort 2001, Section 9.1], this yields $m_{i}=2 i$ and $n_{i}=2 g-2 i+1$ for $1 \leq i \leq g$; also, let

$$
Z_{i}:= \begin{cases}X_{i / 2} & \text { if } i \text { is even, } \\ Y_{c-(i-1) / 2} & \text { if } i \text { is odd. }\end{cases}
$$

By [Oort 2001, Section 9.1], for $1 \leq i \leq g$, the action of $F$ is given by $F\left(Y_{i}\right)=0$ and $F\left(X_{i}\right)=Z_{i}$ and the action of $V$ by $V\left(Z_{i}\right)=0$ and $V\left(Z_{2 g-i+1}\right)=(-1)^{i-1} Y_{i}$.

More notation is needed to give an explicit description of $D\left(G_{c}\right)$.

Notation 5.9. Let $c \in \mathbb{N}$ be fixed. Let $I:=\{j \in \mathbb{N} \mid\lceil(c+1) / 2\rceil \leq j \leq c\}$, which is a set of cardinality $\lfloor(c+1) / 2\rfloor$. For $j \in I$, let $\ell(j)$ be the odd part of $j$, and let $e(j)$ be the nonnegative integer such that $j=2^{e(j)} \ell(j)$. Let $s(j):=c-(\ell(j)-1) / 2$. One can check that $\{s(j) \mid j \in I\}=I$. Also, let $m(j):=2 c-2 j+1$, and let $\epsilon(j)$ be the nonnegative integer such that $t(j):=2^{\epsilon(j)} m(j) \in I$. One can check that $\{t(j) \mid j \in I\}=I$. Thus, there is a unique bijection $\iota: I \rightarrow I$ such that $t(\iota(j))=s(j)$ for each $j \in I$.

Proposition 5.10. Recall Notation 5.9. For $c \in \mathbb{N}$, the set $\left\{X_{j} \mid j \in I\right\}$ generates the Dieudonné module $D\left(G_{c}\right)$ as an $\mathbb{E}$-module subject to the relations, for $j \in I$, $F^{e(j)+1}\left(X_{j}\right)+V^{\epsilon(\iota(j))+1}\left(X_{\iota(j)}\right)=0$. Also, $\left\{X_{j} \mid j \in I\right\}$ is a basis for the quotient of $D\left(G_{c}\right)$ by the left ideal $D\left(G_{c}\right)(F, V)$. 
Proof. Proposition 5.8 implies that $F^{e(j)}\left(X_{j}\right)=X_{\ell(j)}$ and $F\left(X_{\ell(j)}\right)=Y_{s(j)}$. Also, $V\left(X_{j}\right)=-Y_{m(j)}$, and so $V^{\epsilon(j)+1}\left(X_{j}\right)=-Y_{t(j)}$. This yields the stated relations. To complete the first claim, it suffices to show that the span of $\left\{X_{j} \mid j \in I\right\}$ under the action of $F$ and $V$ contains the $k$-module generators of $D\left(C_{c}\right)$ listed in Proposition 5.8. This follows from the observations that $X_{i}=F\left(X_{2 i}\right)$ if $1 \leq i \leq\lfloor c / 2\rfloor$, that $Y_{i}=V\left(Y_{i / 2}\right)$ if $i$ is even, and that $Y_{i}=V\left(-X_{c-(i-1) / 2}\right)$ if $i$ is odd. By [Li and Oort 1998, 5.2.8], the dimension of $D\left(G_{c}\right)$ modulo $D\left(G_{c}\right)(F, V)$ equals the $a$-number. Since $a=|I|$ by Corollary 5.3, it follows that the set $|I|$ of generators of $D\left(G_{c}\right)$ is linearly independent modulo $D\left(G_{c}\right)(F, V)$.

Here are some more examples. The columns of the table below list the value of $c$, the generators of $D\left(G_{c}\right)$ as an $\mathbb{E}$-module $\left(X_{i_{1}}-X_{i_{2}}\right.$ denotes $\left.\left\{X_{i} \mid i_{1} \leq i \leq i_{2}\right\}\right)$, and the relations among these generators. The last column is the number of summands of $D\left(G_{c}\right)$ in its decomposition as an $\mathbb{E}$-module (as opposed to as a polarized $\mathbb{E}$-module). The table can be verified in two ways: first, by checking it with Proposition 5.10 and second, by computing the action of $F$ and $V$ on a $k$-basis for $D\left(G_{c}\right)$, using this to construct a final filtration of $D\left(G_{c}\right)$ stable under $V$ and $F^{-1}$, and then checking that it matches the Ekedahl-Oort type of $G_{c}$. In Example 5.11, we illustrate the second method.

\begin{tabular}{|c|c|c|c|}
\hline$c$ & generators & relations & \# summands \\
\hline 5 & $X_{3}-X_{5}$ & $F X_{3}+V^{3} X_{5}, F^{3} X_{4}+V X_{3}, F X_{5}+V X_{4}$ & 1 \\
\hline 6 & $X_{4}-X_{6}$ & $F^{3} X_{4}+V^{2} X_{5}, F X_{5}+V^{3} X_{6}, F^{2} X_{6}+V X_{4}$ & 1 \\
\hline 7 & $X_{4}-X_{7}$ & $\begin{aligned} F^{3} X_{4}+V X_{4}, & F X_{5}+V X_{5}, \\
& F^{2} X_{6}+V^{2} X_{6}, F X_{7}+V^{3} X_{7}\end{aligned}$ & 4 \\
\hline 8 & $X_{5}-X_{8}$ & $\begin{aligned} F X_{5}+V^{2} X_{7}, & F^{2} X_{6}+V X_{5}, \\
& F X_{7}+V X_{6}, F^{4} X_{8}+V^{4} X_{8}\end{aligned}$ & 2 \\
\hline 9 & $X_{5}-X_{9}$ & $\begin{array}{r}F X_{5}+V X_{6}, F^{2} X_{6}+V^{4} X_{9}, F X_{7}+V^{2} X_{8} \\
F^{4} X_{8}+V X_{5}, F X_{9}+V X_{7}\end{array}$ & 1 \\
\hline 10 & $X_{6}-X_{10}$ & $\begin{array}{r}F^{2} X_{6}+V X_{6}, F X_{7}+V X_{7}, F^{4} X_{8}+V^{2} X_{8} \\
F X_{9}+V^{2} X_{9}, F^{2} X_{10}+V^{4} X_{10}\end{array}$ & 5 \\
\hline
\end{tabular}

Example 5.11. For $c=7$, the group scheme $G_{7}$ that has Ekedahl-Oort type $[0,1,1,2,2,3,3]$ is isomorphic to a direct sum of polarized $\mathrm{BT}_{1}$ group schemes of ranks $p^{2}, p^{4}$, and $p^{8}$ and has Dieudonné module

$$
\mathbf{M}:=\mathbb{E} / \mathbb{E}(F+V) \oplus \mathbb{E} / \mathbb{E}\left(F^{2}+V^{2}\right) \oplus \mathbb{E} / \mathbb{E}\left(V+F^{3}\right) \oplus \mathbb{E} / \mathbb{E}\left(F^{3}+V\right) .
$$

Proof. Let $\left\{1_{A}, V_{A}\right\}$ be the basis of the submodule $A=\mathbb{E} / \mathbb{E}(F+V)$ of $\mathbf{M}$, $\left\{1_{B}, V_{B}, V_{B}^{2}, F_{B}^{2}\right\}$ the basis of the submodule $B=\mathbb{E} / \mathbb{E}\left(F^{2}+V^{2}\right),\left\{1_{C}, V_{C}, V_{C}^{2}, V_{C}^{3}\right\}$ the basis of the submodule $C=\mathbb{E} / \mathbb{E}\left(F+V^{3}\right)$, and $\left\{1_{C^{\prime}}, F_{C^{\prime}}, F_{C^{\prime}}^{2}, F_{C^{\prime}}^{3}\right\}$ the basis of 
the submodule $C^{\prime}=\mathbb{E} / \mathbb{E}\left(F^{3}+V\right)$. The action of Frobenius and Verschiebung on the elements of these bases is

\begin{tabular}{c|cccccccccccccc}
\hline$x$ & $1_{A}$ & $V_{A}$ & $1_{B}$ & $V_{B}$ & $V_{B}^{2}$ & $F_{B}$ & $1_{C}$ & $V_{C}$ & $V_{C}^{2}$ & $V_{C}^{3}$ & $1_{C^{\prime}}$ & $F_{C^{\prime}}$ & $F_{C^{\prime}}^{2}$ & $F_{C^{\prime}}^{3}$ \\
\hline$V x$ & $V_{A}$ & 0 & $V_{B}$ & $V_{B}^{2}$ & 0 & 0 & $V_{C}$ & $V_{C}^{2}$ & $V_{C}^{3}$ & 0 & $F_{C^{\prime}}^{3}$ & 0 & 0 & 0 \\
$F x$ & $V_{A}$ & 0 & $F_{B}$ & 0 & 0 & $V_{B}^{2}$ & $V_{C}^{3}$ & 0 & 0 & 0 & $F_{C^{\prime}}$ & $F_{C^{\prime}}^{2}$ & $F_{C^{\prime}}^{3}$ & 0 \\
\hline
\end{tabular}

To verify the proposition, one can repeatedly apply $V$ and $F^{-1}$ to construct a filtration $N_{1} \subset N_{2} \subset \cdots \subset N_{14}$ of $\mathbf{M}$ as a $k$-vector space that is stable under the action of $V$ and $F^{-1}$ such that $i=\operatorname{dim}\left(N_{i}\right)$. To save space, we summarize the calculation by listing a generator $t_{i}$ for $N_{i} / N_{i-1}$ :

\begin{tabular}{c|cccccccccccccc}
\hline$i$ & 1 & 2 & 3 & 4 & 5 & 6 & 7 & 8 & 9 & 10 & 11 & 12 & 13 & 14 \\
\hline$t_{i}$ & $V_{C}^{3}$ & $V_{C}^{2}$ & $V_{B}^{2}$ & $V_{C}$ & $V_{A}$ & $F_{C^{\prime}}^{3}$ & $V_{B}$ & $1_{C}$ & $F_{C^{\prime}}^{2}$ & $1_{A}$ & $F_{B}$ & $F_{C^{\prime}}$ & $1_{C^{\prime}}$ & $1_{B}$ \\
\hline
\end{tabular}

Then one can check that $V\left(N_{i}\right)=N_{\lfloor i / 2\rfloor}$ and $F^{-1}\left(N_{i}\right)=N_{7+\lceil i / 2\rceil}$, which verifies that the Ekedahl-Oort type of $\mathbf{M}$ is $[0,1,1,2,2,3,3]$.

Remark 5.12. One could ask when $D\left(G_{c}\right)$ decomposes as much as numerically possible, in other words, when the $a$-number equals the number of summands of $D\left(G_{c}\right)$ in its decomposition as an $\mathbb{E}$-module. For example, $D\left(G_{c}\right)$ has this property when $c \in\{1,2,3,4,7,10\}$ but not when $c \in\{5,6,8,9\}$. This phenomenon occurs if and only if the bijection $\iota$ from Notation 5.9 is the identity.

Remark 5.13. The group scheme $G_{8}$ decomposes as the direct sum of two indecomposable polarized $\mathrm{BT}_{1}$ group schemes, one whose Ekedahl-Oort type is $[0,0,1,1]$ and the other whose covariant Dieudonné module is $\mathbb{E} / \mathbb{E}\left(F^{4}+V^{4}\right)$. We take this opportunity to note that there is a mistake in [Pries 2008, Example in Section 3.3]. The covariant Dieudonné module of $I_{4,3}=[0,0,1,1]$ is stated incorrectly. To fix it, consider the method of [Oort 2001, Section 9.1]. Consider the $k$-vector space of dimension 8 generated by $X_{1}, \ldots, X_{4}$ and $Y_{1}, \ldots, Y_{4}$. Consider the operation $F$ defined by $F\left(Y_{i}\right)=0$ for $1 \leq i \leq 4$,

$$
F\left(X_{1}\right)=Y_{4}, \quad F\left(X_{2}\right)=Y_{3}, \quad F\left(X_{3}\right)=X_{1}, \quad F\left(X_{4}\right)=Y_{2} .
$$

Consider the operation $V$ defined by

$$
\begin{array}{llll}
V\left(X_{1}\right)=0, & V\left(X_{2}\right)=-Y_{4}, & V\left(X_{3}\right)=-Y_{2}, & V\left(X_{4}\right)=-Y_{1}, \\
V\left(Y_{1}\right)=Y_{3}, & V\left(Y_{2}\right)=0, & V\left(Y_{3}\right)=0, & V\left(Y_{4}\right)=0 .
\end{array}
$$

Thus, $D\left(I_{4,3}\right)$ is generated by $X_{2}, X_{3}$, and $X_{4}$ modulo the three relations

$$
F X_{2}+V^{2} X_{4}, \quad F^{2} X_{3}+V X_{2}, \quad V X_{3}+F X_{4} .
$$


5c. Newton polygons. There are several results in characteristic 2 about the Newton polygons of hyperelliptic (for example, Artin-Schreier) curves $X$ of genus $g$ and 2rank 0. For example, [Blache 2012, Remark 3.6] states that if $2^{n-1}-1 \leq g \leq 2^{n}-2$, then the generic first slope of the Newton polygon of an Artin-Schreier curve of genus $g$ and 2-rank 0 is $1 / n$. This statement is generalized to odd primes $p$ in [Blache 2012, Proposition 3.5]. See also earlier work in [Scholten and Zhu 2002, Theorem 1.1(III)].

The Ekedahl-Oort type of $J_{X}$ [2] gives information about the Newton polygon of $X$ but does not determine it completely. Using Corollary 5.3 and [Harashita 2007, Section 3.1 and Theorem 4.1], one can show that the first slope of the Newton polygon of $X$ is at least $1 / n$. Since this is weaker than [Blache 2012, Theorem 4.3], we do not include the details.

More generally, one could consider the case that $X$ is a hyperelliptic $k$-curve of genus $g$ and arbitrary $p$-rank. One could use Theorem 5.2 to give partial information (namely, a lower bound) for the Newton polygon of $X$.

\section{Acknowledgments}

We would like to thank Jeff Achter and the anonymous referee for helpful comments.

\section{References}

[Blache 2012] R. Blache, "Valuation of exponential sums and the generic first slope for Artin-Schreier curves”, J. Number Theory 132:10 (2012), 2336-2352. MR 2944758 Zbl 06074389

[Bouw 2001] I. I. Bouw, "The p-rank of ramified covers of curves", Compositio Math. 126:3 (2001), 295-322. MR 2002e:14045 Zbl 1056.14036

[Cartier 1957] P. Cartier, "Une nouvelle opération sur les formes différentielles", C. R. Acad. Sci. Paris 244 (1957), 426-428. MR 18,870b Zbl 0077.04502

[Crew 1984] R. M. Crew, "Etale p-covers in characteristic p", Compositio Math. 52:1 (1984), 31-45. MR 85f:14011 Zbl 0558.14009

[Demazure 1972] M. Demazure, Lectures on p-divisible groups, Lecture Notes in Mathematics 302, Springer, Berlin, 1972. MR 88a:14049 Zbl 0247.14010

[Ekedahl 1987] T. Ekedahl, "On supersingular curves and abelian varieties", Math. Scand. 60:2 (1987), 151-178. MR 88g:14037 Zbl 0641.14007

[Elkin 2011] A. Elkin, "The rank of the Cartier operator on cyclic covers of the projective line", $J$. Algebra 327 (2011), 1-12. MR 2011m:14071 Zbl 1216.14026

[Goren 2002] E. Z. Goren, Lectures on Hilbert modular varieties and modular forms, CRM Monograph Series 14, American Mathematical Society, Providence, RI, 2002. MR 2003c:11038 Zbl 0986.11037

[Harashita 2007] S. Harashita, "Ekedahl-Oort strata and the first Newton slope strata", J. Algebraic Geom. 16:1 (2007), 171-199. MR 2007k:14043 Zbl 1113.14031

[Hardy and Ramanujan 1918] G. H. Hardy and S. Ramanujan, "Asymptotic formulæ in combinatory analysis", Proc. Lond. Math. Soc. (2) 17 (1918), 75-115. Reprinted as pp. 276-309 in Collected papers of Srinivasa Ramanujan, AMS Chelsea Publ., Providence, RI, 2000. JFM 46.0198.02 
[Johnston 2007] O. Johnston, "A note on the $a$-numbers and $p$-ranks of Kummer covers", preprint, 2007. arXiv 0710.2120

[Kraft 1975] H. Kraft, "Kommutative algebraische $p$-Gruppen (mit Anwendungen auf $p$-divisible Gruppen und abelsche Varietäten)", manuscript, Sonderforsch. Bereich Bonn, 1975.

[Li and Oort 1998] K.-Z. Li and F. Oort, Moduli of supersingular abelian varieties, Lecture Notes in Mathematics 1680, Springer, Berlin, 1998. MR 99e:14052 Zbl 0920.14021

[Madden 1978] D. J. Madden, "Arithmetic in generalized Artin-Schreier extensions of $k(x)$ ", $J$. Number Theory 10:3 (1978), 303-323. MR 80d:12009 Zbl 0384.12008

[Moonen 2001] B. Moonen, "Group schemes with additional structures and Weyl group cosets", pp. 255-298 in Moduli of abelian varieties (Texel Island, 1999), edited by C. Faber et al., Progr. Math. 195, Birkhäuser, Basel, 2001. MR 2002c:14074 Zbl 1084.14523

[Nart and Sadornil 2004] E. Nart and D. Sadornil, "Hyperelliptic curves of genus three over finite fields of even characteristic", Finite Fields Appl. 10:2 (2004), 198-220. MR 2005a:11090 Zbl 1049.11063

[Oda 1969] T. Oda, "The first de Rham cohomology group and Dieudonné modules", Ann. Sci. École Norm. Sup. (4) 2 (1969), 63-135. MR 39 \#2775 Zbl 0175.47901

[Oort 2001] F. Oort, "A stratification of a moduli space of abelian varieties", pp. 345-416 in Moduli of abelian varieties (Texel Island, 1999), edited by C. Faber et al., Progr. Math. 195, Birkhäuser, Basel, 2001. MR 2002b:14055 Zbl 1052.14047

[Pries 2008] R. Pries, "A short guide to $p$-torsion of abelian varieties in characteristic p", pp. 121129 in Computational arithmetic geometry (San Francisco, CA, 2006), edited by K. E. Lauter and K. A. Ribet, Contemp. Math. 463, American Mathematical Society, Providence, RI, 2008. MR 2009m:11085 Zbl 1151.14328

[Scholten and Zhu 2002] J. Scholten and H. J. Zhu, "Hyperelliptic curves in characteristic 2", Int. Math. Res. Not. 2002:17 (2002), 905-917. MR 2003d:11089 Zbl 1034.14013

[Serre 1968] J.-P. Serre, Corps locaux, 2nd ed., Actualités scientifiques et industrielles 1296, Hermann, Paris, 1968. MR 50 \#7096 Zbl 0423.12017

[Stichtenoth 2009] H. Stichtenoth, Algebraic function fields and codes, 2nd ed., Graduate Texts in Mathematics 254, Springer, Berlin, 2009. MR 2010d:14034 Zbl 1155.14022

[Subrao 1975] D. Subrao, “The p-rank of Artin-Schreier curves”, Manuscripta Math. 16:2 (1975), 169-193. MR 51 \#12868 Zbl 0321.14017

[Sullivan 1975] F. J. Sullivan, " $p$-torsion in the class group of curves with too many automorphisms", Arch. Math. (Basel) 26 (1975), 253-261. MR 52 \#13846 Zbl 0331.14016

[van der Geer 1999] G. van der Geer, "Cycles on the moduli space of abelian varieties", pp. 65-89 in Moduli of curves and abelian varieties: The Dutch intercity seminar on moduli, edited by C. Faber and E. Looijenga, Aspects Math. E33, Vieweg, Braunschweig, 1999. MR 2001b:14009 Zbl 0974.14031

[Yui 1978] N. Yui, "On the Jacobian varieties of hyperelliptic curves over fields of characteristic $p>2 ”$, J. Algebra 52:2 (1978), 378-410. MR 58 \#10920 Zbl 0404.14008

Communicated by Richard Taylor

Received 2010-07-07 Revised 2012-04-11 Accepted 2012-04-16

A.Elkin@warwick.ac.uk Mathematics Institute, University of Warwick, Coventry, CV4 7AL, United Kingdom

pries@math.colostate.edu

Department of Mathematics, Colorado State University, Fort Collins, CO, 80523, United States 


\section{Algebra \& Number Theory}

msp.org/ant

\section{EDITORS}

MANAGING EDITOR

Bjorn Poonen

Massachusetts Institute of Technology

Cambridge, USA

\author{
EDITORIAL BOARD CHAIR \\ David Eisenbud \\ University of California \\ Berkeley, USA
}

\section{BOARD OF EDITORS}

Georgia Benkart

Dave Benson

Richard E. Borcherds

John H. Coates

J-L. Colliot-Thélène

Brian D. Conrad

Hélène Esnault

Hubert Flenner

Edward Frenkel

Andrew Granville

Joseph Gubeladze

Ehud Hrushovski

Craig Huneke

Mikhail Kapranov

Yujiro Kawamata

János Kollár

Yuri Manin

Barry Mazur

Philippe Michel
University of Wisconsin, Madison, USA

University of Aberdeen, Scotland

University of California, Berkeley, USA

University of Cambridge, UK

CNRS, Université Paris-Sud, France

University of Michigan, USA

Freie Universität Berlin, Germany

Ruhr-Universität, Germany

University of California, Berkeley, USA

Université de Montréal, Canada

San Francisco State University, USA

Hebrew University, Israel

University of Virginia, USA

Yale University, USA

University of Tokyo, Japan

Princeton University, USA

Northwestern University, USA

Harvard University, USA

École Polytechnique Fédérale de Lausanne
Susan Montgomery

Shigefumi Mori

Raman Parimala

Jonathan Pila

Victor Reiner

Karl Rubin

Peter Sarnak

Joseph H. Silverman

Michael Singer

Vasudevan Srinivas

J. Toby Stafford

Bernd Sturmfels

Richard Taylor

Ravi Vakil

Michel van den Bergh

Marie-France Vignéras

Kei-Ichi Watanabe

Efim Zelmanov
University of Southern California, USA

RIMS, Kyoto University, Japan

Emory University, USA

University of Oxford, UK

University of Minnesota, USA

University of California, Irvine, USA

Princeton University, USA

Brown University, USA

North Carolina State University, USA

Tata Inst. of Fund. Research, India

University of Michigan, USA

University of California, Berkeley, USA

Harvard University, USA

Stanford University, USA

Hasselt University, Belgium

Université Paris VII, France

Nihon University, Japan

University of California, San Diego, USA

\section{PRODUCTION}

production@msp.org

Silvio Levy, Scientific Editor

See inside back cover or msp.org/ant for submission instructions.

The subscription price for 2013 is US \$200/year for the electronic version, and \$350/year ( $\$ 40$, if shipping outside the US) for print and electronic. Subscriptions, requests for back issues and changes of subscribers address should be sent to MSP.

Algebra \& Number Theory (ISSN 1944-7833 electronic, 1937-0652 printed) at Mathematical Sciences Publishers, 798 Evans Hall \#3840, c/o University of California, Berkeley, CA 94720-3840 is published continuously online. Periodical rate postage paid at Berkeley, CA 94704, and additional mailing offices.

ANT peer review and production are managed by EditFLOW ${ }^{\circledR}$ from Mathematical Sciences Publishers.

\section{PUBLISHED BY}

- mathematical sciences publishers

nonprofit scientific publishing

http://msp.org/

(C) 2013 Mathematical Sciences Publishers 


\section{Algebra \& Number Theory}

Volume $7 \quad$ No. $3 \quad 2013$

Ekedahl-Oort strata of hyperelliptic curves in characteristic 2

ARSEN ELKIN and RACHEL PRIES

Cycle classes and the syntomic regulator

Bruno Chiarellotto, Alice Ciccioni and Nicola Mazzari

Zeros of real irreducible characters of finite groups

SELENA MARINELli and PHAM HUU TIEP

The biHecke monoid of a finite Coxeter group and its representations

FLORENT HIVERT, ANNE SCHILLING and NICOLAS THIÉRY

Shuffle algebras, homology, and consecutive pattern avoidance

VLADIMIR DOTSENKO and ANTON KHOROSHKIN

701

Preperiodic points for families of polynomials

Dragos Ghioca, Liang-Chung Hsia and Thomas J. Tucker

$F$-blowups of normal surface singularities

NobUo HaRa, TADAKaZU SAWADA and TAKEHIKo YASUdA 